\title{
PRESIONES DINÁMICAS DEL SUELO EN LUMBRERAS
}

\author{
Javier Avilés López ${ }^{(1)}$ y Luis Eduardo Pérez Rocha ${ }^{(2)}$
}

\begin{abstract}
RESUMEN
Se presenta un método de frontera para calcular las presiones del suelo y las fuerzas asociadas producidas por sismo en lumbreras profundas de gran diámetro. La respuesta del sistema ante incidencia vertical de ondas de cortante se construye mediante expansiones de modos de ondas usando el método del estrato delgado. La solución satisface rigurosamente las condiciones de compatibilidad (desplazamientos y fuerzas) entre suelo y lumbrera, así como las condiciones de superficie libre y basamento rígido. Para sistemas típicos, se evalúa la importancia relativa de la flexibilidad del muro y la condición de apoyo; se construye además un conjunto de tablas de diseño para calcular las distribuciones del cortante y momento con la profundidad.
\end{abstract}

Palabras clave: Presiones dinámicas del suelo, interacción suelo-estructura, fuerza cortante, momento flexionante, respuesta sísmica

\begin{abstract}
A boundary method to compute the soil pressures and the associated forces produced by earthquakes in deeply embedded large-diameter shafts is presented. The system response to vertically propagating shear waves is constructed by means of wave mode expansions using the thin layer method. The solution fulfills rigorously the compatibility conditions (displacements and forces) between soil and shaft, as well as the free-surface and rigid-bedrock conditions. For seismically excited shafts, the relative importance of the wall flexibility and the supporting condition is evaluated; a set of design tables to compute the shear and moment distributions with depth are also constructed.
\end{abstract}

Key Words: Soil dynamic pressure, soil-structure interaction, shear force, bending moment, earthquake response.

\section{INTRODUCCIÓN}

Con el propósito de incrementar la capacidad de desalojo de aguas residuales y pluviales en el Valle de México, se está construyendo el túnel emisor oriente que inicia en la intersección de las Avs. Gran Canal y Río de los Remedios, Ciudad de México, y llega al portal de salida en el Ejido de Conejos, Estado de Hidalgo. La gran obra consta de un túnel de $7 \mathrm{~m}$ de diámetro y $62 \mathrm{~km}$ de longitud, más 24 lumbreras de acceso con profundidades desde 32 hasta $150 \mathrm{~m}$ y diámetros desde 12 hasta $20 \mathrm{~m}$. Si bien las estructuras

Artículo recibido el 15 de marzo de 2010 y aprobado para su publicación el 4 de septiembre de 2011

(1) Instituto Mexicano de Tecnología del Agua, Paseo Cuahnahuac 8532, Jiutepec 62550, javiles@tlaloc.imta.mx

(2) Instituto de Investigaciones Eléctricas, Reforma 113, Cuernavaca, Morelos 62490, lepr@iie.org.mx 
subterráneas son menos vulnerables a los movimientos sísmicos que las estructuras superficiales, es necesario garantizar la seguridad de la obra ante los movimientos del terreno esperados en sitios del trazo con características geotécnicas muy diferentes.

Los efectos sísmicos en estructuras subterráneas se evalúan con enfoques diferentes a los utilizados para estructuras superficiales. En general, las estructuras superficiales se diseñan para las fuerzas de inercia originadas por el movimiento de su base. En cambio, las acciones de diseño para estructuras subterráneas se expresan en términos de las deformaciones impuestas a la estructura por el movimiento del suelo. Para túneles, los criterios de diseño sísmico están bien establecidos (Hashash et al, 2001; St. John y Zahrah, 1987). Para lumbreras, sin embargo, los métodos de análisis sísmico son escasos y limitados.

El enfoque de diseño más sencillo es el que ignora la interacción de la lumbrera con el suelo circundante. Según este enfoque, primero se estiman las deformaciones del suelo en campo libre y luego la lumbrera se diseña para acomodarse a estas deformaciones. El resultado es aceptable cuando las rigideces de ambos elementos son similares. En caso contrario, es necesario considerar los efectos de interacción debidos al contraste de rigidez entre suelo y lumbrera. Estos efectos adquieren relevancia en sitios con capas alternadas de suelo blando y duro, en cuyas interfases se concentran los momentos flexionantes generados ante el paso de las ondas sísmicas. Zeevaert (1983) ha propuesto un método seudoestático para el cálculo de fuerzas sísmicas en lumbreras. Aunque ignora el efecto de la frecuencia de excitación en la inercia del suelo y la consideración de la excitación sísmica es aproximada, el método es adecuado para el análisis de lumbreras poco profundas.

Durante sismos intensos, las lumbreras sufren significativas curvaturas debidas a las deformaciones del suelo. Entre las pocas soluciones reportadas en la literatura para calcular los momentos flexionantes causados por el movimiento lateral está la de Tajimi (1969), basada en la hipótesis de desplazamientos verticales nulos, así como las de Veletsos y Younan (1994; 1995), basadas en la hipótesis de esfuerzos verticales nulos. La suposición de esfuerzos verticales nulos, introducida por Arias et al (1981) en muros de retención, es superior a la de desplazamientos verticales nulos en suelos con relación de Poisson grande. La principal limitación de estas soluciones es la consideración de estructuras rígidas con base fija. Para estas condiciones, las acciones del suelo resultan ser excesivamente grandes respecto a las que se tendrían en lumbreras flexibles flotantes. La flexibilidad de la estructura y la traslación-rotación de su base tienen como efecto una reducción notable de dichas acciones.

En este trabajo se presenta un método de frontera para análisis sísmico dinámico de sistemas suelolumbrera. El modelo consiste de un cilindro elástico enterrado en un depósito de suelo con base rígida, formando dos regiones: una interior con la lumbrera y el suelo de soporte y otra exterior con el suelo circundante. Para cada región, los desplazamientos y fuerzas se expresan mediante superposición de modos de ondas que se propagan horizontalmente. Dichos modos se calculan con el método del estrato delgado, de suerte que la solución es discreta en la dirección vertical y continua en la horizontal. En la interfase entre las dos regiones, se imponen las condiciones de frontera de compatibilidad de desplazamientos y fuerzas. Como excitación del sistema se considera la incidencia vertical de ondas de cortante.

Aplicando el método propuesto, se examinan los efectos de la flexibilidad del muro y la condición de apoyo. Asimismo, se calculan los valores estáticos del cortante y momento a diferente profundidad, así como los promedios de valores máximos debidos a excitación sísmica. El cociente entre ellos permite definir factores de amplificación que consideran el efecto dinámico del suelo. Estos resultados se presentan en forma de tablas de diseño para diferentes contrastes de rigidez suelo/estructura y relaciones de esbeltez estructural. Para ilustrar el cálculo del cortante y momento, se analiza el caso de un ejemplo práctico. 


\section{FORMULACIÓN DEL PROBLEMA}

\section{Ecuaciones de movimiento}

En la fig. 1 se muestra el sistema suelo-lumbrera investigado. Sean $u, v$ y $w$ los desplazamientos radial, tangencial y axial, respectivamente, en coordenadas cilíndricas $(r, \theta, z)$. Si el sistema se subdivide en $N$ estratos horizontales, las ecuaciones diferenciales que gobiernan el movimiento armónico en el estrato $1 \leq j \leq N$ son

$\nabla^{2} u-\frac{u}{r^{2}}-\frac{2}{r^{2}} \frac{\partial v}{\partial \theta}+\frac{1}{1-2 v_{j}} \frac{\partial \varepsilon}{\partial r}+\frac{\omega^{2}}{\beta_{j}^{2}} u=0$

$\nabla^{2} v-\frac{v}{r^{2}}-\frac{2}{r^{2}} \frac{\partial u}{\partial \theta}+\frac{1}{1-2 v_{j}} \frac{1}{r} \frac{\partial \varepsilon}{\partial \theta}+\frac{\omega^{2}}{\beta_{j}^{2}} v=0$

$\nabla^{2} w+\frac{1}{1-2 v_{j}} \frac{\partial \varepsilon}{\partial z}+\frac{\omega^{2}}{\beta_{j}^{2}} w=0$

donde $\omega$ es la frecuencia de excitación, $v_{j}$ la relación de Poisson y $\beta_{j}=G_{j} / \rho_{j}$ la velocidad de ondas

de corte, siendo $G_{j}$ el módulo de cortante y $\rho_{j}$ la densidad; $\nabla^{2}$ y $\varepsilon$ son el Laplaciano y la dilatación, respectivamente, definidos como

$\nabla^{2}=\frac{\partial^{2}}{\partial r^{2}}+\frac{1}{r} \frac{\partial}{\partial r}+\frac{1}{r^{2}} \frac{\partial^{2}}{\partial \theta^{2}}+\frac{\partial^{2}}{\partial z^{2}}$

$\varepsilon=\frac{\partial u}{\partial r}+\frac{1}{r}\left(u+\frac{\partial v}{\partial \theta}\right)+\frac{\partial w}{\partial z}$

Los componentes de esfuerzo sobre una superficie cilíndrica están relacionados con los componentes de desplazamiento mediante:

$\sigma_{r}=\lambda_{j} \varepsilon+2 G_{j} \frac{\partial u}{\partial r}$

$\tau_{r z}=G_{j}\left(\frac{\partial u}{\partial z}+\frac{\partial w}{\partial r}\right)$

$\tau_{r \theta}=G_{j}\left(\frac{\partial v}{\partial r}-\frac{v}{r}+\frac{1}{r} \frac{\partial u}{\partial \theta}\right)$

donde $\lambda_{j}=2 v_{j} G_{j} /\left(1-2 v_{j}\right)$ es la constante de Lamé. 


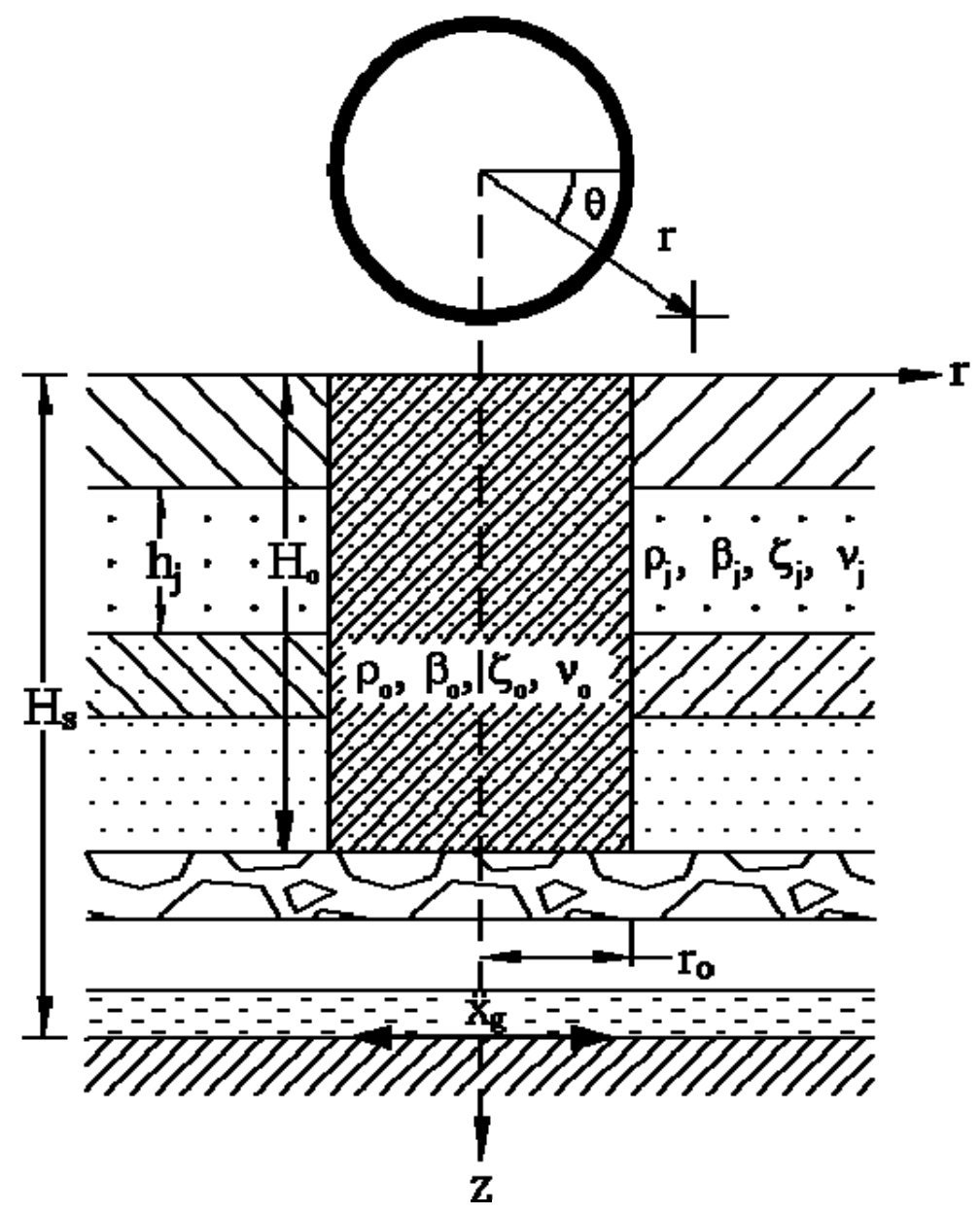

Figura 1. Sistema suelo-lumbrera sujeto a excitación horizontal.

\section{Descomposición azimutal}

La simetría axial de la estructura permite realizar una descomposición azimutal de la solución. Según Kausel y Roësset (1975 y 1977), los desplazamientos modales (radial, vertical y tangencial) pueden obtenerse mediante separación de variables como se indica a continuación.

\section{Modos generalizados de Rayleigh en deformación plana:}

$$
\begin{aligned}
& u(r, \theta, z)=u(r, z)\left\{\begin{array}{c}
\cos n \theta \\
\operatorname{sen} n \theta
\end{array}\right\}, \quad \operatorname{con} u=k U(z) C_{n}^{\prime}(k r) \\
& w(r, \theta, z)=w(r, z)\left\{\begin{array}{c}
\cos n \theta \\
\operatorname{sen} n \theta
\end{array}\right\}, \quad \operatorname{con} w=-i k W(z) C_{n}(k r)
\end{aligned}
$$


$v(r, \theta, z)=\nabla(r, z)\left\{\begin{array}{r}-\operatorname{sen} n \theta \\ \cos n \theta\end{array}\right\}, \quad \operatorname{con} \nabla=\frac{n}{r} U(z) C_{n}(k r)$

\section{Modos generalizados de Love en cortante antiplano:}

$u(r, \theta, z)=u(r, z)\left\{\begin{array}{c}\cos n \theta \\ \operatorname{sen} n \theta\end{array}\right\}, \quad$ con $u=\frac{n}{r} V(z) C_{n}(k r)$

$w(r, \theta, z)=0$

$v(r, \theta, z)=\nabla(r, z)\left\{\begin{array}{r}-\operatorname{sen} n \theta \\ \cos n \theta\end{array}\right\}, \quad \operatorname{con} \nabla=k V(z) C_{n}^{\prime}(k r)$

donde $k$ es el número de onda horizontal y $n$ el número de onda azimutal; $C_{n}(\xi)$ es una solución de la ecuación de Bessel de orden $n$, dada por

$C_{n}^{\prime \prime}+\frac{1}{\xi} C_{n}^{\prime}+\left(1-\frac{n^{2}}{\xi^{2}}\right) C_{n}=0$

El factor armónico en el tiempo $e^{i \omega t}$ se ha omitido por simplicidad. Para modos simétricos con respecto al plano $\theta=0, u$ y $w$ se combinan $\operatorname{con} \cos n \theta$ y $\nabla \operatorname{con}-\operatorname{sen} n \theta$; en cambio, $u$ y $w$ se combinan $\operatorname{con} \operatorname{sen} n \theta$ y $\nabla \operatorname{con} \cos n \theta$ para modos antisimétricos. Sustituyendo las ecs. 9-14 en las ecs. 68 , se puede verificar que

$$
\begin{aligned}
& \sigma_{r}(r, \theta, z)=\bar{\sigma}_{r}(r, z)\left\{\begin{array}{c}
\cos n \theta \\
\operatorname{sen} n \theta
\end{array}\right\} \rightarrow f_{r}(r, \theta, z)=\int \sigma_{r}(r, \theta, z) d z=f_{r}(r, z)\left\{\begin{array}{c}
\cos n \theta \\
\operatorname{sen} n \theta
\end{array}\right\} \\
& \tau_{r z}(r, \theta, z)=\bar{\tau}_{r z}(r, z)\left\{\begin{array}{c}
\cos n \theta \\
\operatorname{sen} n \theta
\end{array}\right\} \rightarrow f_{z}(r, \theta, z)=\int \tau_{r z}(r, \theta, z) d z=f_{z}(r, z)\left\{\begin{array}{c}
\cos n \theta \\
\operatorname{sen} n \theta
\end{array}\right\} \\
& \tau_{r \theta}(r, \theta, z)=\bar{\tau}_{r \theta}(r, z)\left\{\begin{array}{r}
-\operatorname{sen} n \theta \\
\cos n \theta
\end{array}\right\} \rightarrow f_{\theta}(r, \theta, z)=\int \tau_{r \theta}(r, \theta, z) d z=f_{\theta}(r, z)\left\{\begin{array}{r}
-\operatorname{sen} n \theta \\
\cos n \theta
\end{array}\right\}
\end{aligned}
$$

donde $\bar{f}_{r}(r, z)=\int \sigma_{r}(r, z) d z, \bar{f}_{z}(r, z)=\int \tau_{r z}(r, z) d z$ y $\bar{f}_{\theta}(r, z)=\int \tau_{r \theta}(r, z) d z$. De esta forma se demuestra que la descomposición azimutal de esfuerzos y fuerzas es idéntica a la de desplazamientos. La selección de la función azimutal, $\cos n \theta$ o sen $n \theta$, depende de la física del problema. Para excitación horizontal $x_{g}$, sus componentes en coordenadas cilíndricas son $u_{g}=x_{g} \cos \theta, w_{g}=0 \quad$ y $\quad v_{g}=-x_{g} \operatorname{sen} \theta$. En consecuencia, sólo se requiere del análisis de vibraciones simétricas para el número azimutal $n=1$. Así, el problema tridimensional se reduce a uno bidimensional en el plano $r-z$. 


\section{Condiciones de frontera}

Para fines de análisis, el domino en estudio se divide en dos regiones: 1) una interior $i\left(r \leq r_{o}, 0 \leq z \leq H_{s}\right)$ para la lumbrera y el suelo de soporte y 2$)$ otra exterior $e\left(r \geq r_{o}, 0 \leq z \leq H_{s}\right)$ para el suelo circundante, siendo $r_{o}$ el radio de la lumbrera y $H_{s}$ la profundidad del depósito de suelo. Es interesante hacer notar que la región exterior está formada sólo por estratos de suelo, mientras que la región interior está formada por estratos tanto de suelo como de lumbrera. Para satisfacer las condiciones de frontera del problema, se utiliza el método de colocación en puntos nodales. En cada región, los campos de desplazamientos y fuerzas nodales pueden construirse mediante la superposición del campo libre y de un campo difractado, como sigue:

$$
\begin{aligned}
& \widetilde{\delta}_{\varepsilon}=\widetilde{\delta}_{\varepsilon}^{f}+\widetilde{\delta}_{\varepsilon}^{d}=\widetilde{\delta}_{\varepsilon}^{f}+\widetilde{\Delta}_{\varepsilon} \widetilde{C}_{\varepsilon}, \quad \varepsilon=i, e \\
& \tilde{f}_{\varepsilon}=\widetilde{f}_{\varepsilon}^{f}+\widetilde{f}_{\varepsilon}^{d}=\widetilde{f}_{\varepsilon}^{f}+\widetilde{F}_{\varepsilon} \widetilde{C}_{\varepsilon}, \quad \varepsilon=i, e
\end{aligned}
$$

donde: $\quad \widetilde{\delta}_{\varepsilon}^{f}=$ vector de desplazamientos de campo libre en la región $\varepsilon$

$\tilde{f}_{\varepsilon}^{f}=$ vector de fuerzas de campo libre en la región $\varepsilon$

$\widetilde{\delta}_{r}^{d}=$ vector de desplazamientos difractados en la región $\varepsilon$

$\tilde{f}_{\varepsilon}^{d}=$ vector de fuerzas difractadas en la región $\varepsilon$

$\widetilde{\Delta}_{\varepsilon}=$ matriz de desplazamientos modales en la región $\varepsilon$

$\tilde{F}_{r}=$ matriz de fuerzas modales en la región $\varepsilon$

$\widetilde{C}_{\varepsilon}=$ vector de coeficientes de participación en la región $\varepsilon$

Las condiciones de frontera que deben satisfacerse en la interfase entre las dos regiones son la compatibilidad de desplazamientos y fuerzas nodales, esto es:

$\widetilde{\delta}_{e}\left(r_{o}, z_{j}\right)=\widetilde{\delta}_{i}\left(r_{o}, z_{j}\right), \quad 1 \leq j \leq N$

$$
\tilde{f}_{e}\left(r_{o}, z_{j}\right)=\tilde{f}_{i}\left(r_{o}, z_{j}\right), \quad 1 \leq j \leq N
$$

Sustituyendo las ecs. 19 y 20 en las ecs. 21 y 22, respectivamente, se llega al sistema matricial de ecuaciones algebraicas

$$
\left[\begin{array}{l|l}
\widetilde{\Delta}_{e}\left(r_{o}, z_{j}\right) & -\widetilde{\Delta}_{i}\left(r_{o}, z_{j}\right) \\
\hline \widetilde{F}_{e}\left(r_{o}, z_{j}\right) & -\widetilde{F}_{i}\left(r_{o}, z_{j}\right)
\end{array}\right]\left\{\begin{array}{l}
\widetilde{C}_{e} \\
\frac{\widetilde{C}_{i}}{i}
\end{array}\right\}=\left\{\begin{array}{l}
\widetilde{\delta}_{i}^{f}\left(r_{o}, z_{j}\right)-\widetilde{\delta}_{e}^{f}\left(r_{o}, z_{j}\right) \\
\widetilde{f}_{i}^{f}\left(r_{o}, z_{j}\right)-\widetilde{f}_{e}^{f}\left(r_{o}, z_{j}\right)
\end{array}\right\}, \quad 1 \leq j \leq N
$$

Resolviendo este sistema complejo de ecuaciones algebraicas, se obtienen los coeficientes de participación que definen los campos de desplazamientos y fuerzas nodales en ambas regiones.

Cabe señalar que la lumbrera se ha modelado como un cilindro sólido, que en realidad es hueco. Para considerar este efecto, se pueden utilizar propiedades equivalentes que resultan de igualar la rigidez al corte y la masa del cilindro sólido con los valores de un cilindro hueco. Así se obtienen el módulo de cortante y la densidad equivalentes 


$$
\begin{aligned}
& G_{c}^{*}=G_{c}\left(1-\frac{r_{1}^{2}}{r_{2}^{2}}\right) \\
& \rho_{c}^{*}=\rho_{c}\left(1-\frac{r_{1}^{2}}{r_{2}^{2}}\right)
\end{aligned}
$$

donde $G_{c}$ y $\rho_{c}$ son el módulo de cortante y la densidad del material, mientras que $r_{1}$ y $r_{2}$ son los radios interior y exterior del muro. Nótese que $G_{c}^{*}=G_{c}$ y $\rho_{c}^{*}=\rho_{c}$ para la losa de fondo, ya que ahí $r_{1}=0$. Para cualquier valor de $r_{1} / r_{2}<1$, se tiene que $\beta_{c}^{*}=\beta_{c}$. En lumbreras de sección telescopiada pueden usarse los promedios ponderados de $r_{1}$ y $r_{2}$ como radios equivalentes.

\section{IMPLEMENTACIÓN NUMÉRICA}

\section{Campos difractados}

Aplicando el método del estrato delgado (Lysmer y Waas, 1972; Lysmer y Drake, 1972), puede demostrarse que las eigenfunciones discretas $U\left(z_{j}\right)$ y $W\left(z_{j}\right)$ con eigenvalor $k$ que satisfacen las ecuaciones de movimiento en deformación plana, las condiciones de continuidad de esfuerzos y desplazamientos entre estratos y las condiciones de frontera de superficie libre y base rígida, se obtienen resolviendo el problema algebraico de valores característicos

$\left[k^{2} \tilde{A}+i k \widetilde{B}+\widetilde{G}-\omega^{2} \tilde{M}\right] \tilde{\Lambda}=\tilde{0}$

donde

$\tilde{\Lambda}=\left\{\begin{array}{c}\Lambda_{2 j-1}=U_{j} \\ \Lambda_{2 j}=W_{j}\end{array}\right\}, \quad 1 \leq j \leq N$

es un eigenvector de amplitudes nodales y $\widetilde{A}, \widetilde{B}, \widetilde{G}$ y $\tilde{M}$ son matrices de $2 N \times 2 N$ ensambladas con las matrices de estrato dadas en el Apéndice I para elementos en deformación plana. De aquí se obtienen los valores de $k_{l}$ y $\tilde{\Lambda}_{l}, 1 \leq l \leq 2 N$, correspondientes a modos generalizados de Rayleigh.

Igualmente puede demostrarse que la eigenfunción discreta $V\left(z_{j}\right)$ con eigenvalor $k$ que satisface la ecuación de movimiento en cortante antiplano, las condiciones de continuidad de esfuerzo y desplazamiento entre estratos y las condiciones de frontera de superficie libre y base rígida, se obtiene resolviendo el problema algebraico de valores característicos

$\left[k^{2} \tilde{A}+\widetilde{G}-\omega^{2} \tilde{M}\right] \tilde{\Lambda}=\widetilde{0}$

donde 


$$
\widetilde{\Lambda}=\left\{\Lambda_{j}=V_{j}\right\}, \quad 1 \leq j \leq N
$$

es un eigenvector de amplitudes nodales y $\widetilde{A}, \widetilde{G}$ y $\tilde{M}$ son matrices de $N \times N$ ensambladas con las matrices de estrato dadas en el Apéndice I para elementos en cortante antiplano. De aquí se obtienen los valores de $k_{l}$ y $\tilde{\Lambda}_{l}, 1 \leq l \leq N$, correspondientes a modos generalizados de Love.

Una vez resueltos los problemas de valores característicos en deformación plana y cortante antiplano, la matriz de desplazamientos modales en la superficie cilíndrica $r=r_{o}$ se construye como

$$
\widetilde{\Delta}_{\varepsilon}=\left[\begin{array}{rl}
\Delta_{3 j-2, l} & =U_{l}\left(r_{o}, z_{j}\right) \\
\Delta_{3 j-1, l} & =w_{l}\left(r_{o}, z_{j}\right) \\
\Delta_{3 j, l} & =\nabla_{l}\left(r_{o}, z_{j}\right)
\end{array}\right], \quad 1 \leq j \leq N \quad \text { y } 1 \leq l \leq 3 N
$$

donde:

$$
\begin{aligned}
& \left.\begin{array}{rl}
\Delta_{3 j-2, l} & =k_{l} U_{j}^{l} C_{n}^{\prime}\left(k_{l} r_{o}\right) \\
\Delta_{3 j-1, l} & =-i k_{l} W_{j}^{l} C_{n}\left(k_{l} r_{o}\right) \\
\Delta_{3 j, l} & =\frac{n}{r_{o}} U_{j}^{l} C_{n}\left(k_{l} r_{o}\right)
\end{array}\right\}, \quad \text { si } 1 \leq l \leq 2 N \\
& \left.\begin{array}{rl}
\Delta_{3 j-2, l} & =\frac{n}{r_{o}} V_{j}^{l-2 N} C_{n}\left(k_{l} r_{o}\right) \\
\Delta_{3 j-1, l} & =0 \\
\Delta_{3 j, l} & =k_{l} V_{j}^{l-2 N} C_{n}^{\prime}\left(k_{l} r_{o}\right)
\end{array}\right\}, \quad \text { si } 2 N+1 \leq l \leq 3 N
\end{aligned}
$$

Los eigenvalores $k_{1}, \ldots, k_{2 N}$ y $k_{2 N+1}, \ldots, k_{3 N}$ corresponden a modos generalizados de Rayleigh y Love, respectivamente. La condición de radiación en la región exterior se cumple usando $C_{n}\left(k_{l} r\right)=H_{n}^{2}\left(k_{l} r\right)$, que es la función de Hankel de segunda especie y orden $n$. Como se requieren modos evanescentes que decaigan con la distancia, éstos se seleccionan tal que $\operatorname{Im}\left[k_{l}\right]<0$. Por otra parte, en la región interior debe usarse $C_{n}\left(k_{l} r\right)=J_{n}\left(k_{l} r\right)$, que es la función de Bessel de primera especie y orden $n$. Para modos estacionarios, la selección del signo de $k_{l}$ es irrelevante; sólo se requiere que los modos correspondientes sean linealmente independientes.

Las fuerzas nodales equivalentes en la superficie cilíndrica $r=r_{o}$ pueden obtenerse integrando los esfuerzos correspondientes con respecto a $z$, usando funciones de forma lineales. Estas fuerzas discretizadas están en equilibrio estático con los esfuerzos de estrato. Según Kausel y Roësset (1975 y 1977), el vector de fuerzas nodales consistentes para el modo $1 \leq l \leq 3 N$ es 


$$
\widetilde{F}_{l}=\left\{\begin{array}{r}
F_{3 j-2}=\bar{f}_{r}\left(r_{o}, z_{j}\right) \\
F_{3 j-1}=\bar{f}_{z}\left(r_{o}, z_{j}\right) \\
F_{3 j}=\bar{f}_{\theta}\left(r_{o}, z_{j}\right)
\end{array}\right\}_{1 \leq j \leq N}=\left\{k_{l}^{2} \widetilde{A}_{\Psi_{l}}+k_{l}[\widetilde{D}-\widetilde{E}+n \tilde{N}] \widetilde{\phi}_{l}-n\left[\frac{n+1}{2} \widetilde{L}+\widetilde{Q}\right] \widetilde{\psi}_{l}\right\}
$$

donde $\widetilde{A}, \widetilde{D}, \widetilde{E}, \widetilde{N}, \widetilde{L}$ y $\widetilde{Q}$ son matrices de $3 N \times 3 N$ ensambladas con las matrices de estrato dadas en el Apéndice I para elementos axisimétricos, mientras que $\widetilde{\psi}_{l}$ y $\widetilde{\phi}_{l}$ son las columnas de las siguientes matrices:

$\widetilde{\psi}=\left[\begin{array}{l}\psi_{3 j-2, l} \\ \psi_{3 j-1, l} \\ \psi_{3 j, l}\end{array}\right], \quad 1 \leq j \leq N \quad$ y $1 \leq l \leq 3 N$

donde:

$$
\begin{aligned}
& \psi_{3 j-2, l}=-U_{j}^{l} C_{n}\left(k_{l} r_{o}\right) \\
& \left.\begin{array}{rl}
\psi_{3 j-1, l} & =-i W_{j}^{l} C_{n-1}\left(k_{l} r_{o}\right) \\
\psi_{3 j, l} & =0
\end{array}\right\}, \quad \text { si } 1 \leq l \leq 2 N \\
& \left.\begin{array}{rl}
\psi_{3 j-2, l} & =0 \\
\psi_{3 j-1, l} & =0 \\
\psi_{3 j, l} & =-V_{j}^{l-2 N} C_{n}\left(k_{l} r_{o}\right)
\end{array}\right\}, \quad \text { si } 2 N+1 \leq l \leq 3 N
\end{aligned}
$$

y

$\widetilde{\phi}=\left[\begin{array}{l}\phi_{3 j-2, l} \\ \phi_{3 j-1, l} \\ \phi_{3 j, l}\end{array}\right], \quad 1 \leq j \leq N \quad y \quad 1 \leq l \leq 3 N$

donde:

$$
\left.\begin{array}{rl}
\phi_{3 j-2, l} & =U_{j}^{l} C_{n-1}\left(k_{l} r_{o}\right) \\
\phi_{3 j-1, l} & =i W_{j}^{l} C_{n}\left(k_{l} r_{o}\right) \\
\phi_{3 j, l} & =0
\end{array}\right\}, \quad \text { si } 1 \leq l \leq 2 N
$$


Los vectores $\widetilde{F}_{l}, 1 \leq l \leq 3 N$, que se obtienen con la ec. 33 son las columnas de la matriz de fuerzas modales

$$
\widetilde{F}_{\varepsilon}=\left[\begin{array}{llll}
\widetilde{F}_{1}, & \widetilde{F}_{2}, & \cdots & \widetilde{F}_{3 N}
\end{array}\right]
$$

Cabe mencionar que la función de onda $C_{n}(\xi)$ que se use para fuerzas modales es la misma que para desplazamientos modales.

\section{Campos libres}

En coordenadas cilíndricas, los desplazamientos de campo libre debidos a la incidencia vertical de ondas de cortante se expresan como

$$
\left.\begin{array}{l}
u(r, \theta, z)=u(r, z) \cos \theta \\
w(r, \theta, z)=0 \\
v(r, \theta, z)=-\nabla(r, z) \operatorname{sen} \theta
\end{array}\right\}
$$

con $u(r, z)=\nabla(r, z)=V(z)$. Siguiendo la formulación de Tassoulas y Kausel (1983) para propagación vertical, $k=0$, las amplitudes nodales $V\left(z_{j}\right)$ se obtienen del sistema de ecuaciones algebraicas

$\left[\widetilde{G}-\omega^{2} \tilde{M}\right] \tilde{V}=\tilde{0}$

donde $\tilde{V}=\left\{V_{j}\right\}, 1 \leq j \leq N+1$. Para resolverlo es necesario imponer el movimiento $V_{N+1}=x_{g}$ en la base, eliminando el último renglón y columna de las matrices $\widetilde{G}$ y $\tilde{M}$ y el último elemento de los vectores $\tilde{V}$ y $\tilde{0}$.

Finalmente, con los desplazamientos de campo libre

$$
\widetilde{\delta}_{\varepsilon}^{f}=\left\{\begin{array}{c}
\delta_{3 j-2}=V_{j} \\
\delta_{3 j-1}=0 \\
\delta_{3 j}=V_{j}
\end{array}\right\}, \quad 1 \leq j \leq N
$$

se obtienen las fuerzas de campo libre

$$
\tilde{f}_{\varepsilon}^{f}=\widetilde{D} \widetilde{\delta}_{\varepsilon}^{f}
$$

donde $\widetilde{D}$ es la matriz usada en la ec. 33 multiplicando las columnas $3 j-1$ por menos uno. Debido a la estructura de esta matriz, las fuerzas radial y tangencial son nulas para excitación horizontal.

\section{Fuerza cortante y momento flexionante}

Establecidas las fuerzas nodales $\bar{f}_{r}^{j}$ y $\bar{f}_{\theta}^{j}$ en la superficie cilíndrica $r=r_{o}$, la fuerza resultante en dirección de la excitación se determina integrando con respecto a $\theta$, esto es: 


$$
F_{x}^{j}=\int_{0}^{2 \pi}\left(f_{r}^{j} \cos ^{2} \theta+f_{\theta}^{j} \operatorname{sen}^{2} \theta\right) r_{o} d \theta=\pi r_{o}\left(f_{r}^{j}+f_{\theta}^{j}\right), \quad 1 \leq j \leq N
$$

Nótese que las fuerzas nodales normal y cortante contribuyen en la misma proporción al empuje lateral. A la profundidad $z$, el cortante $Q_{z}$ y momento $M_{z}$ debidos a las fuerzas $F_{x}^{j}$ pueden calcularse por simple estática.

\section{RESULTADOS NUMÉRICOS}

\section{Excitación armónica}

La validación del modelo numérico se hizo con las soluciones analíticas de Veletsos y Younan (1994; 1995) para un cilindro rígido apoyado en la base de un estrato homogéneo. Se calcularon los valores estáticos de la fuerza cortante $\widetilde{Q}_{o}$ y el momento flexionante $\tilde{M}_{o}$ basales, normalizados con respecto a $\pi r_{o} \rho_{s} x_{g} H_{o}^{2}$ y $\pi r_{o} \rho_{s} x_{g} H_{o}^{3}$, respectivamente. Los valores estáticos corresponden a una excitación armónica cuya frecuencia es mucho menor que la frecuencia fundamental del estrato. Si la base del estrato se considera fija, la excitación estática se representa por fuerzas de cuerpo laterales de intensidad $-\rho_{s} x_{g}$ para el suelo $y-\rho_{o} X_{g}$ para la estructura. Los efectos resultantes no deben confundirse con los efectos estáticos debidos a cargas de gravedad.
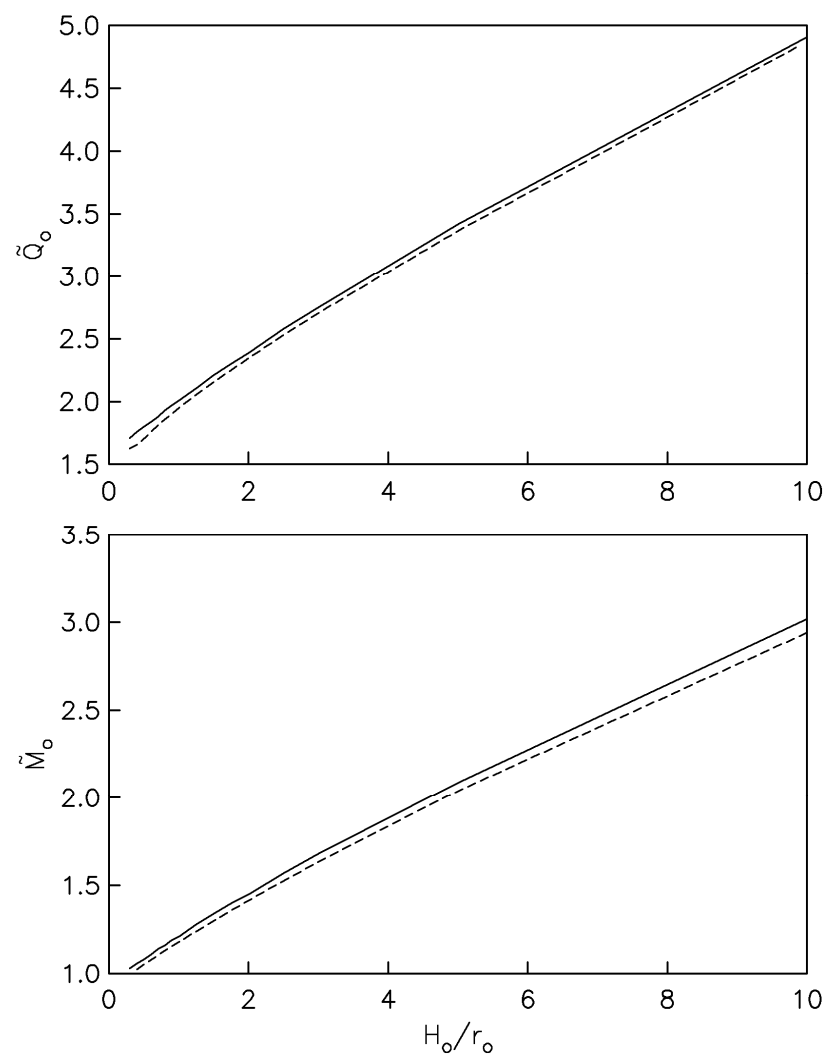

Figura 2. Comparación de valores estáticos del cortante y momento basales obtenidos numéricamente (línea discontinua) contra resultados analíticos (línea continua). 
En la fig. 2 se muestra la variación del cortante y momento basales con la relación de esbeltez $H_{o} / r_{o}$, para un depósito de suelo con $v_{s}=1 / 3$ y $\zeta_{s}=0.05$. La comparación de los resultados numéricos con los cálculos analíticos muestra una excelente concordancia. Puede verse que las acciones del suelo crecen aproximadamente de forma lineal con $H_{o} / r_{o}$, alcanzándose valores inadmisibles para diseño en lumbreras profundas. De ahí la necesidad de incluir la flexibilidad de la estructura.
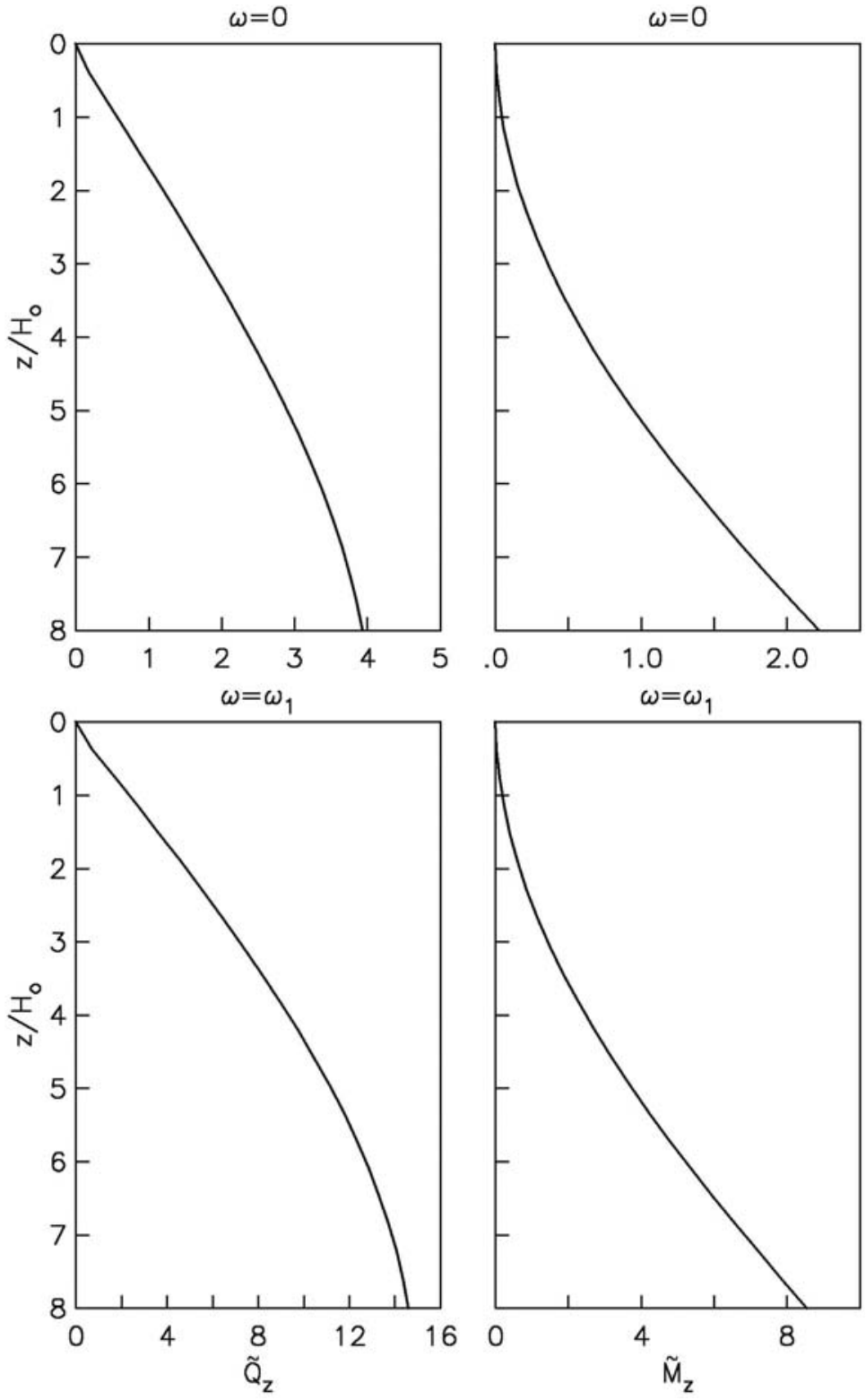

Figura 3. Distribución del cortante y momento con la profundidad para las condiciones estática $(\omega=0)$ y de resonancia $\left(\omega=\omega_{1}\right)$; lumbrera rígida fija en su base. 

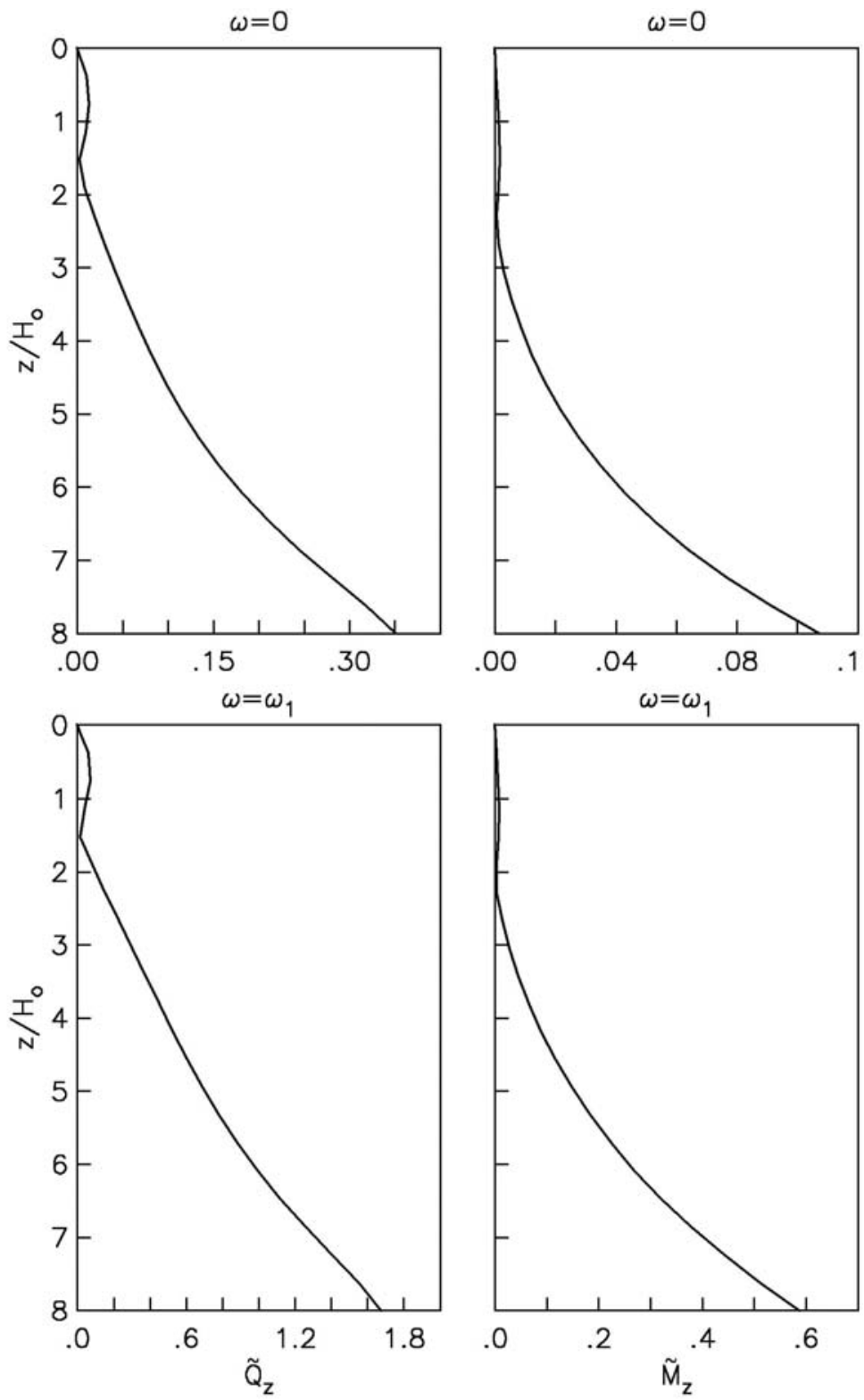

Figura 4. Distribución del cortante y momento con la profundidad para las condiciones estática $(\omega=0)$ y de resonancia ( $\omega=\omega_{1}$ ); lumbrera flexible fija en su base.

En las figs. 3 y 4 se muestran distribuciones de $\widetilde{Q}_{z}$ y $\tilde{M}_{z}$ con la profundidad normalizada $z / H_{o}$, para los casos de lumbrera rígida $\left(\beta_{s} / \beta_{o}=0\right)$ y lumbrera flexible $\left(\beta_{s} / \beta_{o}=0.2\right)$, respectivamente. Se han considerado los siguientes valores de los parámetros del sistema: $H_{o} / r_{o}=H_{s} / r_{o}=8, v_{o}=0.25, v_{s}=0.45$ y $\zeta_{o}=\zeta_{s}=0.05$; el espesor del muro es de $1 \mathrm{~m}$. Los resultados que se grafican son para las frecuencias de excitación $\omega=0$ (respuesta estática) y $\omega_{1}=\pi \beta_{s} / 2 H_{s}$ (respuesta resonante). Se observa que el efecto de la flexibilidad del muro reduce extraordinariamente la magnitud del cortante y momento, a la vez que altera su distribución con la profundidad debido a la participación de los modos superiores del suelo. Como las respuestas estática y resonante tienen formas muy parecidas, es de esperarse un comportamiento similar para la respuesta sísmica. 

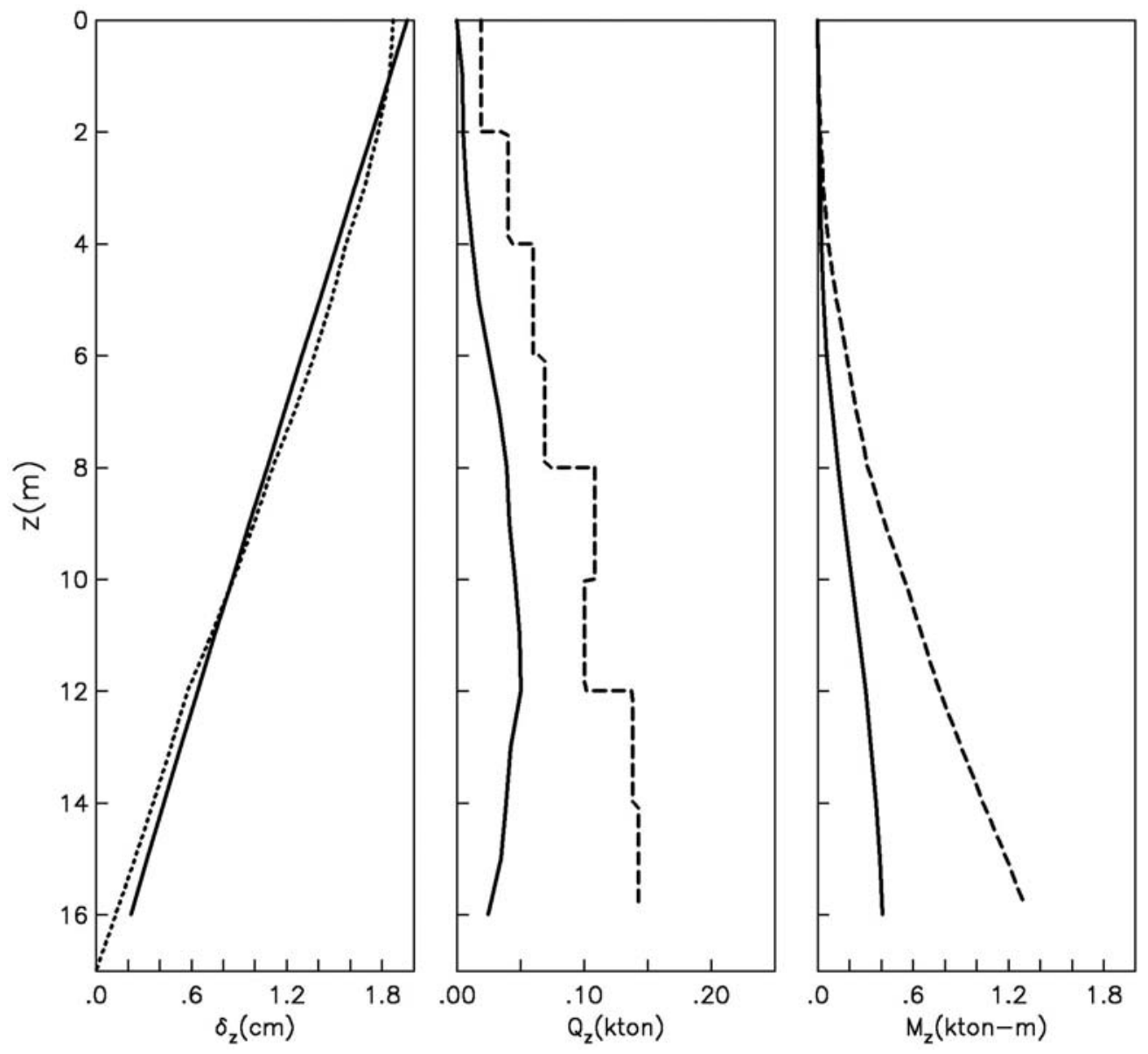

Figura 5. Variación con la profundidad del desplazamiento, cortante y momento en una lumbrera flotante; respuesta de campo libre (….) y solución de Zeevaert (-----).

\section{Excitación transitoria}

Con un método seudo-estático, Zeevaert (1983) analizó una lumbrera de $4 \mathrm{~m}$ de diámetro y $16 \mathrm{~m}$ de profundidad embebida en un medio estratificado de $17 \mathrm{~m}$ de espesor y $0.95 \mathrm{~s}$ de periodo dominante. El muro de la lumbrera es de $0.3 \mathrm{~m}$ de espesor y su base está conectada a un resorte rotacional de $6.36 \times 10^{6}$ ton- $\mathrm{m} / \mathrm{rad}$, que simula la rigidez del ducto horizontal donde se apoya. La consideración de la excitación sísmica se hizo de forma aproximada, especificando la aceleración del suelo en la superficie y estimando la configuración de desplazamientos laterales usada como excitación. Para una aceleración de $1 \mathrm{~m} / \mathrm{s}^{2}$ en la superficie, se obtuvieron los resultados que se describen a continuación. Estos se comparan con los calculados para diferentes condiciones de apoyo mediante el método propuesto.

En nuestro caso, la excitación se modeló con acelerogramas sintéticos que cumplen en promedio con el espectro de peligro uniforme en roca, escalados de forma que se reproduzca la aceleración especificada en la superficie. Con el propósito de mostrar la importancia de la condición de apoyo, se analizaron tres casos: 1) $H_{o}=16 \mathrm{~m}$, lumbrera flotante; 2) $H_{o}=17 \mathrm{~m}$, lumbrera de punta; y $H_{o}=18 \mathrm{~m}$, lumbrera empotrada. Se supuso que el basamento tiene una velocidad de ondas de cortante igual a 500 $\mathrm{m} / \mathrm{s}$. Para cada una de estos casos, los resultados obtenidos se presentan en las figs. 5, 6 y 7 , respectivamente. 

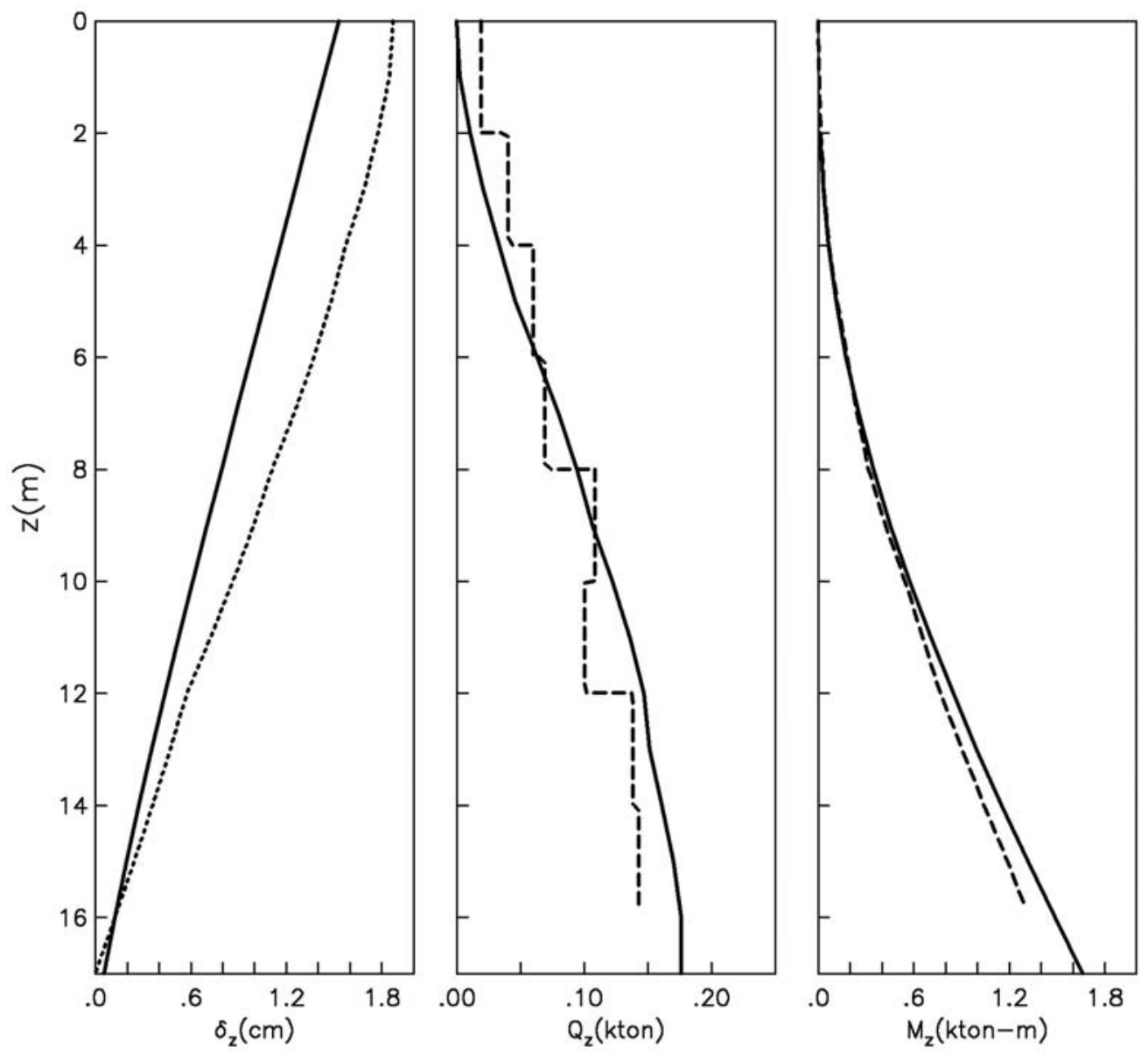

Figura 6. Variación con la profundidad del desplazamiento, cortante y momento en una lumbrera de punta; respuesta de campo libre (….) y solución de Zeevaert (-----).

Obsérvese que el desplazamiento relativo de la lumbrera flotante tiende a seguir el desplazamiento del terreno y así se desarrollan los menores elementos mecánicos. Para los otros casos se reduce significativamente el desplazamiento relativo de la lumbrera, debido a la restricción del movimiento de su base al apoyarse o enterrarse en el basamento. La reducción del desplazamiento se traduce en un incremento de las acciones del suelo comparadas con las generadas en la lumbrera flotante. Este incremento es mayor a medida que se rigidiza la condición de apoyo. La solución de Zeevaert se ajusta muy bien a los resultados de la lumbrera de punta.

\section{Tablas de diseño}

Aplicando el método propuesto, se analizaron los sistemas suelo-lumbrera indicados en la tabla 1, usando un modelo geotécnico del suelo formado por dos capas: el estrato superior representa los depósitos superficiales, donde se presenta la mayor amplificación de la respuesta sísmica, mientras que el estrato inferior representa los depósitos profundos. Para la excitación sísmica, se simularon acelerogramas sintéticos compatibles con el espectro de peligro uniforme en roca para 475 años de periodo de retorno. Este nivel de diseño corresponde a 10\% de probabilidad de excedencia durante 50 años de vida útil de la obra, recomendable para estructuras importantes. 

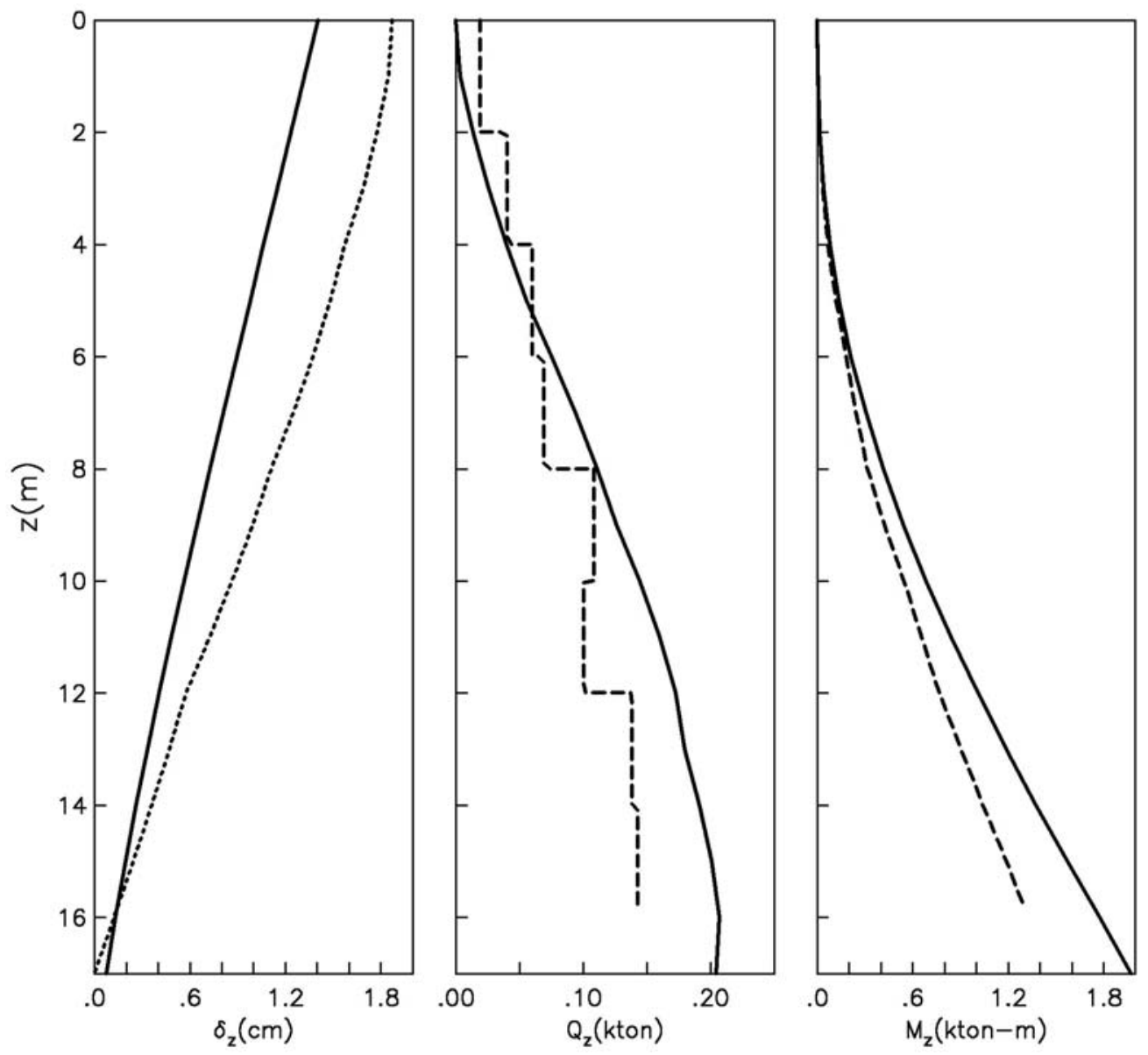

Figura 7. Variación con la profundidad del desplazamiento, cortante y momento en una lumbrera empotrada; respuesta de campo libre (…..) y solución de Zeevaert (-----).

Los parámetros del sistema que se han fijado constantes son los siguientes:

- $\quad$ Lumbrera:

$v_{o}=0.2, \quad \zeta_{o}=0.05$

- Depósitos superficiales:

$v_{1}=0.45, \quad \zeta_{1}=0.1$

- Depósitos profundos:

$v_{2}=0.3, \quad \zeta_{2}=0.08$

- $\quad$ Relación de densidades:

$\rho_{o} / \rho_{1}=1.5, \quad \rho_{1} / \rho_{2}=0.8$

Tabla 1. Parámetros de los sistemas investigados.

\begin{tabular}{ccccccc}
\hline \multicolumn{2}{c}{ Caso } & $H_{o} / r_{o}$ & $H_{1} / r_{o}$ & $H_{2} / r_{o}$ & $\beta_{o} / \beta_{1}$ & $\beta_{1} / \beta_{2}$ \\
\hline $\mathrm{A}$ & 1 & 9 & 4 & 6 & 4.5 & 0.4 \\
& 2 & & & & 9 & 0.333 \\
& 3 & & & & 18 & 0.25 \\
\hline $\mathrm{B}$ & 1 & 12 & 4 & 9 & 4.5 & 0.4 \\
& 2 & & & & 9 & 0.333 \\
& 3 & & & & 18 & 0.25 \\
\hline $\mathrm{C}$ & 1 & 15 & 4 & 12 & 4.5 & 0.4 \\
& 2 & & & & 9 & 0.333 \\
& 3 & & & & 18 & 0.25 \\
\hline
\end{tabular}


Tabla 2. Coeficientes de respuesta para el caso A1.

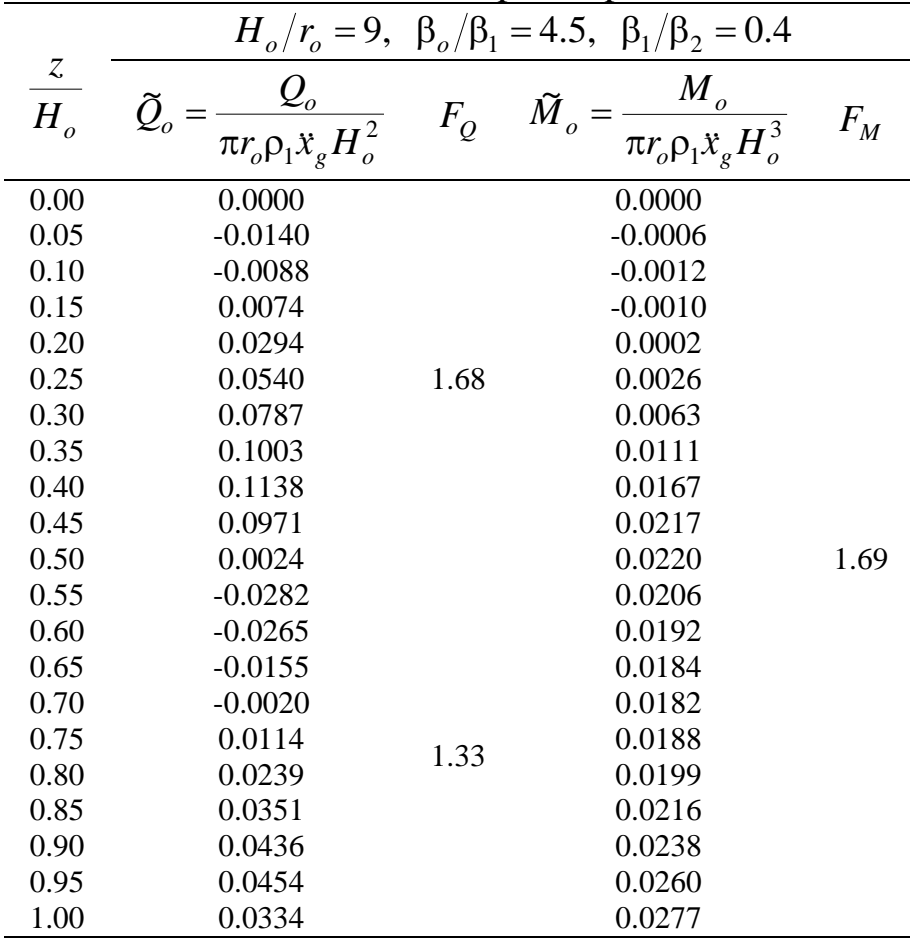
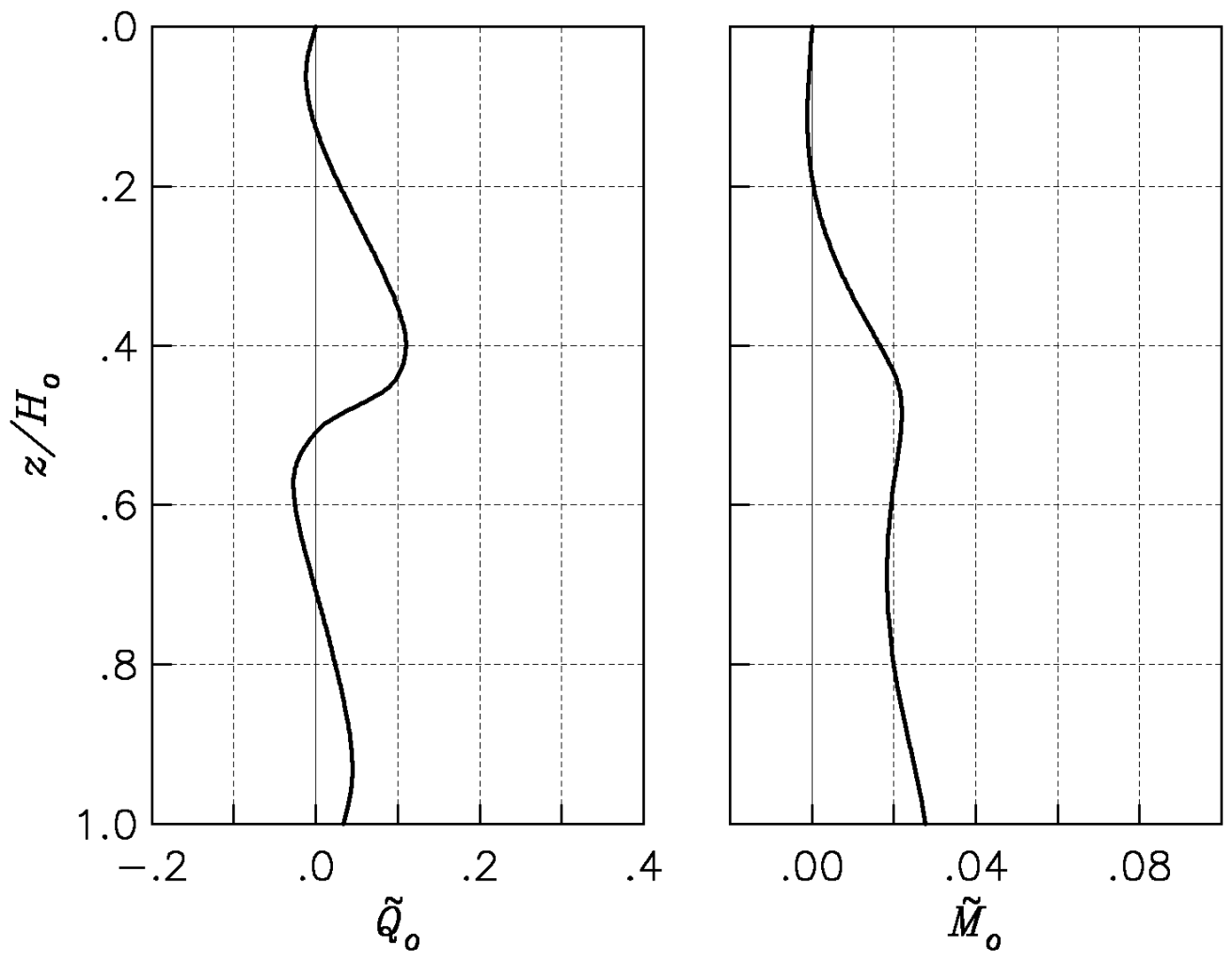
Tabla 3. Coeficientes de respuesta para el caso A2.

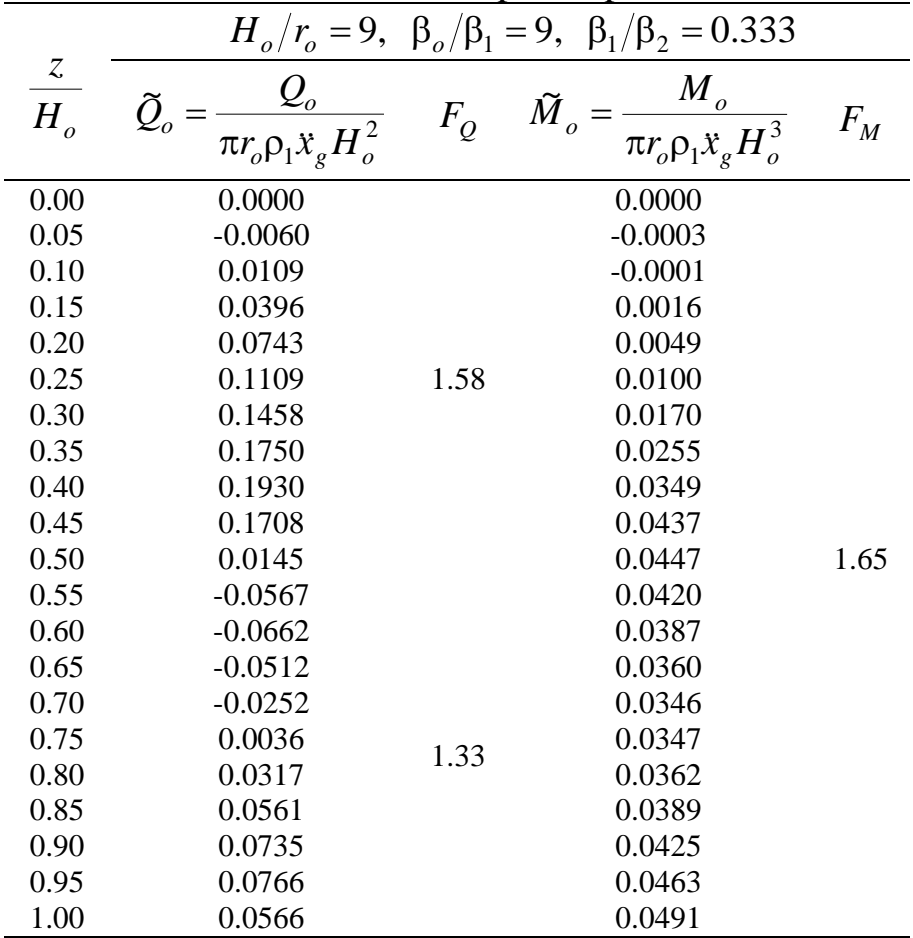
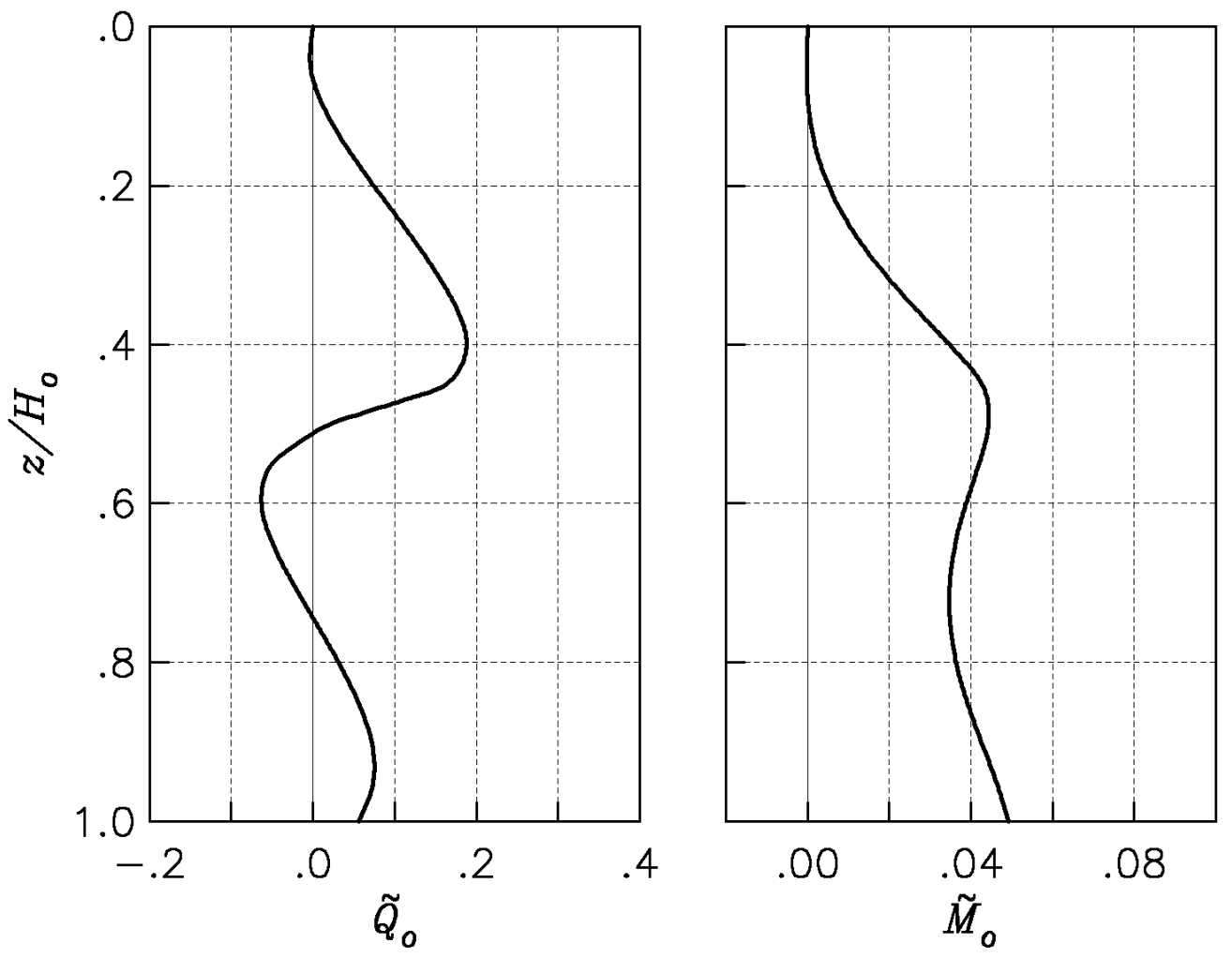
Tabla 4. Coeficientes de respuesta para el caso A3.

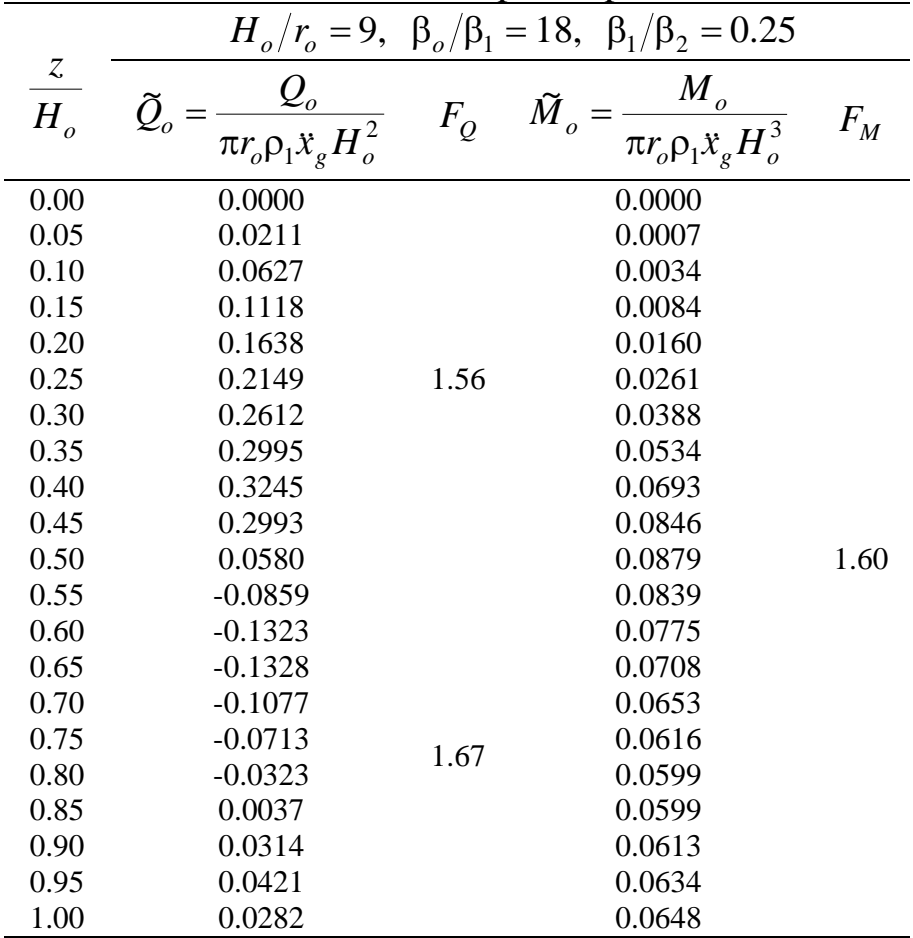
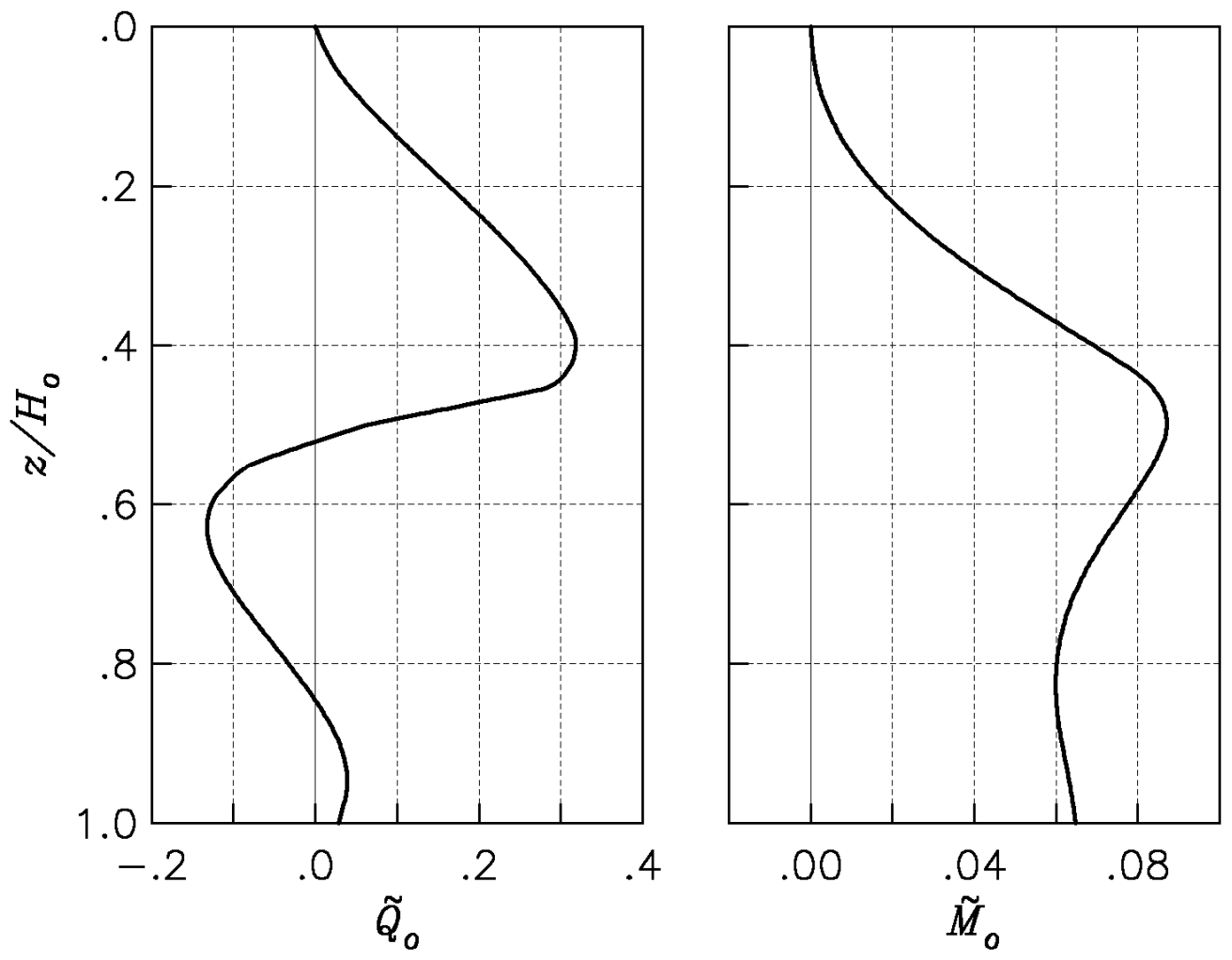
Tabla 5. Coeficientes de respuesta para el caso B1.

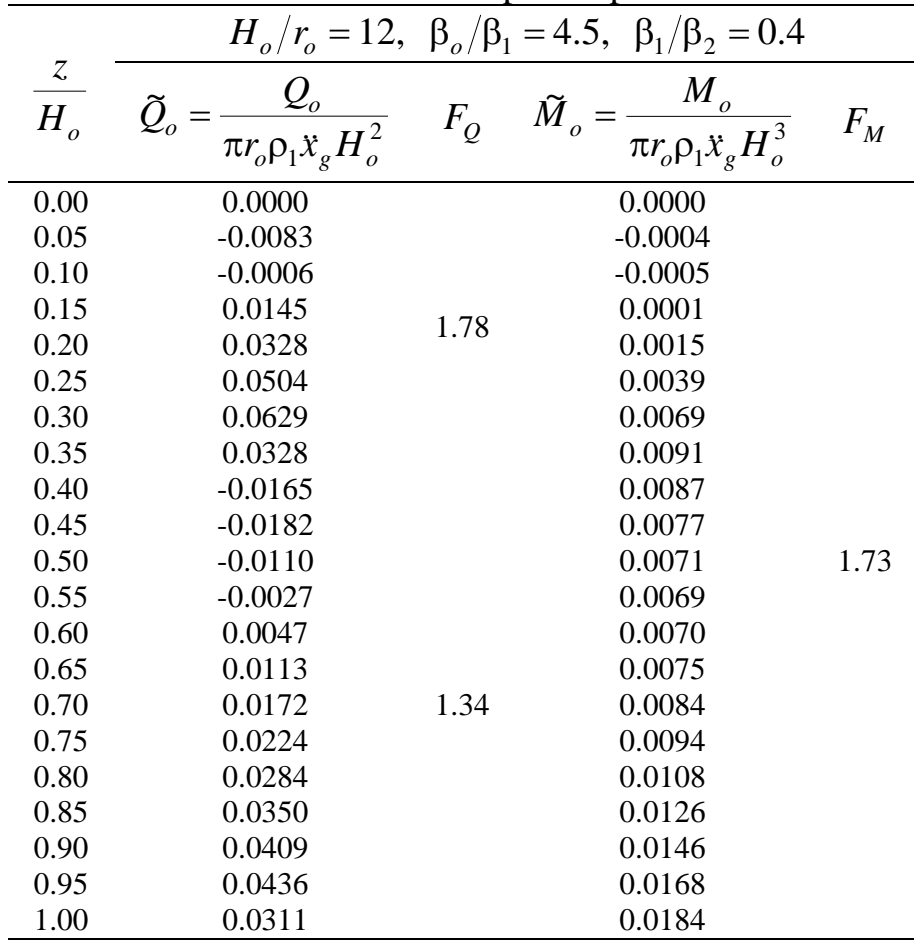
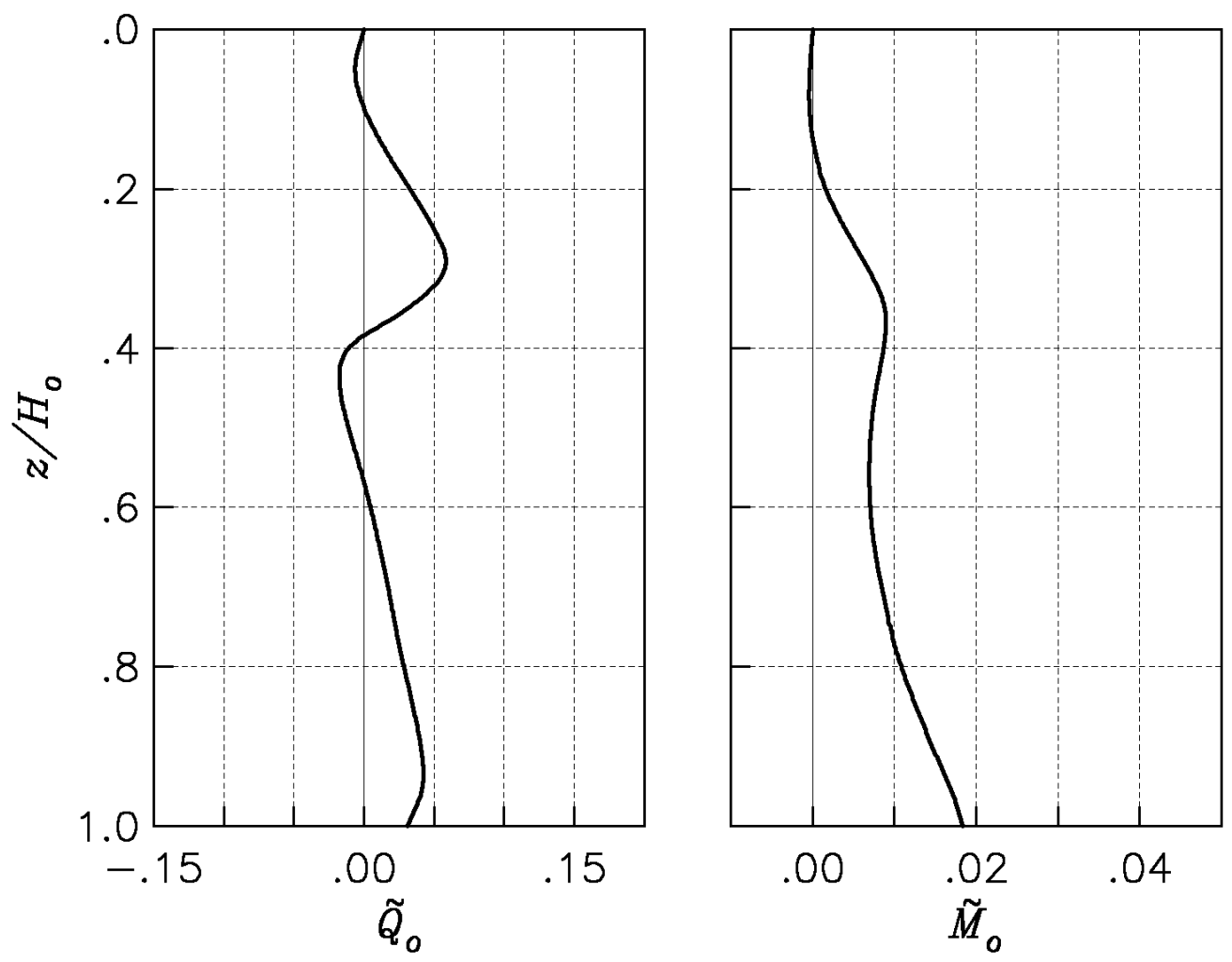
Tabla 6. Coeficientes de respuesta para el caso B2.

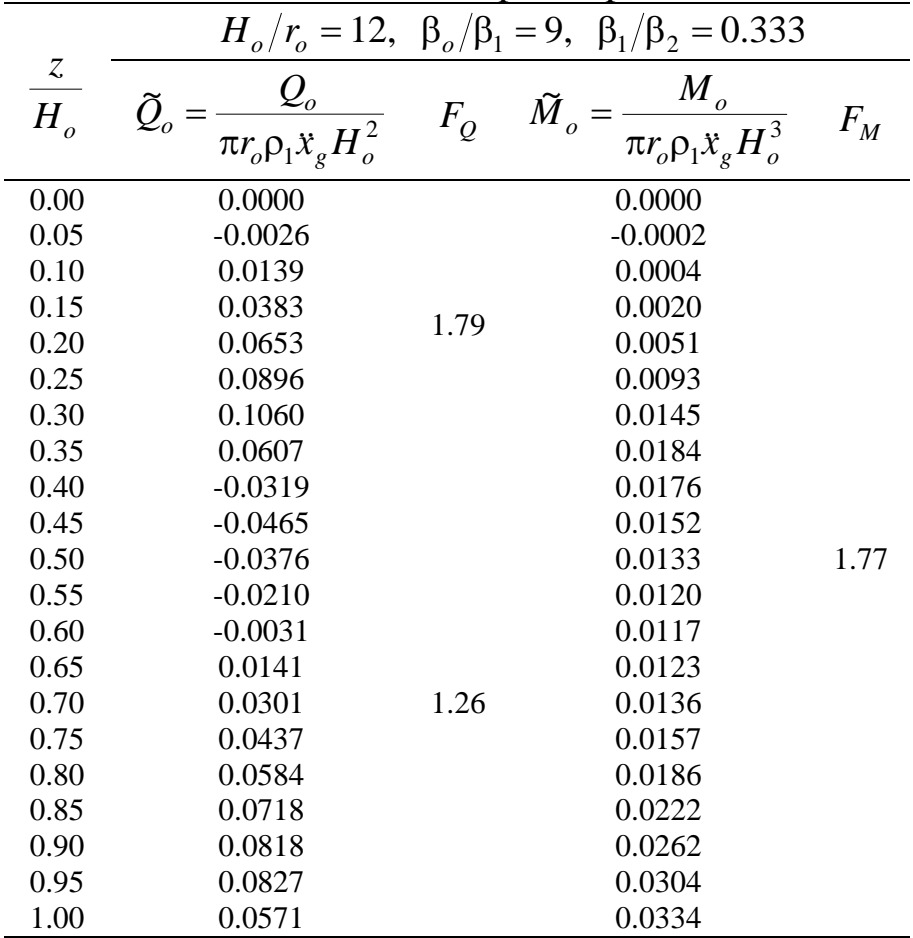
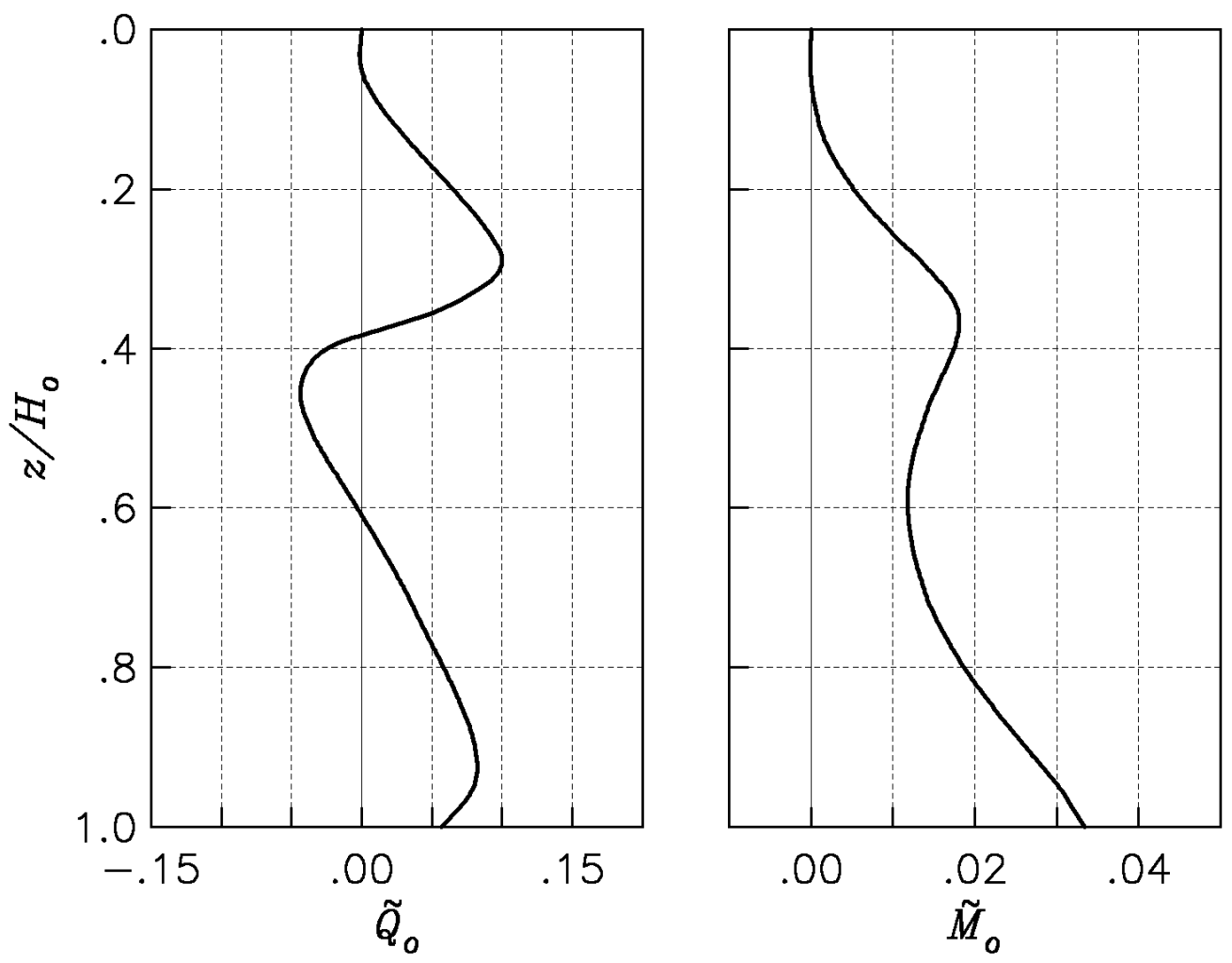
Tabla 7. Coeficientes de respuesta para el caso B3.

\begin{tabular}{lcccc}
\hline & \multicolumn{3}{c}{$H_{o} / r_{o}=12, \beta_{o} / \beta_{1}=18, \beta_{1} / \beta_{2}=0.25$} \\
\cline { 5 - 5 }$H_{o}$ & $\tilde{Q}_{o}=\frac{Q_{o}}{\pi r_{o} \rho_{1} \chi_{g} H_{o}^{2}}$ & $F_{Q}$ & $\tilde{M}_{o}=\frac{M_{o}}{\pi r_{o} \rho_{1} x_{g} H_{o}^{3}}$ & $F_{M}$ \\
\hline 0.00 & 0.0000 & & 0.0000 & \\
0.05 & 0.0155 & & 0.0006 & \\
0.10 & 0.0486 & & 0.0027 & \\
0.15 & 0.0865 & 1.72 & 0.0066 & \\
0.20 & 0.1240 & & 0.0125 & \\
0.25 & 0.1561 & & 0.0200 & \\
0.30 & 0.1782 & & 0.0287 & \\
0.35 & 0.1150 & & 0.0357 & \\
0.40 & -0.0441 & & 0.0350 & \\
0.45 & -0.0916 & & 0.0307 & \\
0.50 & -0.0933 & & 0.0260 & 1.78 \\
0.55 & -0.0743 & & 0.0220 & \\
0.60 & -0.0463 & & 0.0195 & \\
0.65 & -0.0160 & & 0.0183 & \\
0.70 & 0.0140 & 1.21 & 0.0188 & \\
0.75 & 0.0394 & & 0.0206 & \\
0.80 & 0.0650 & & 0.0238 & \\
0.85 & 0.0862 & 0.0281 & \\
0.90 & 0.0996 & & 0.0330 & \\
0.95 & 0.0985 & & 0.0380 & \\
1.00 & 0.0647 & & 0.0415 & \\
\hline
\end{tabular}
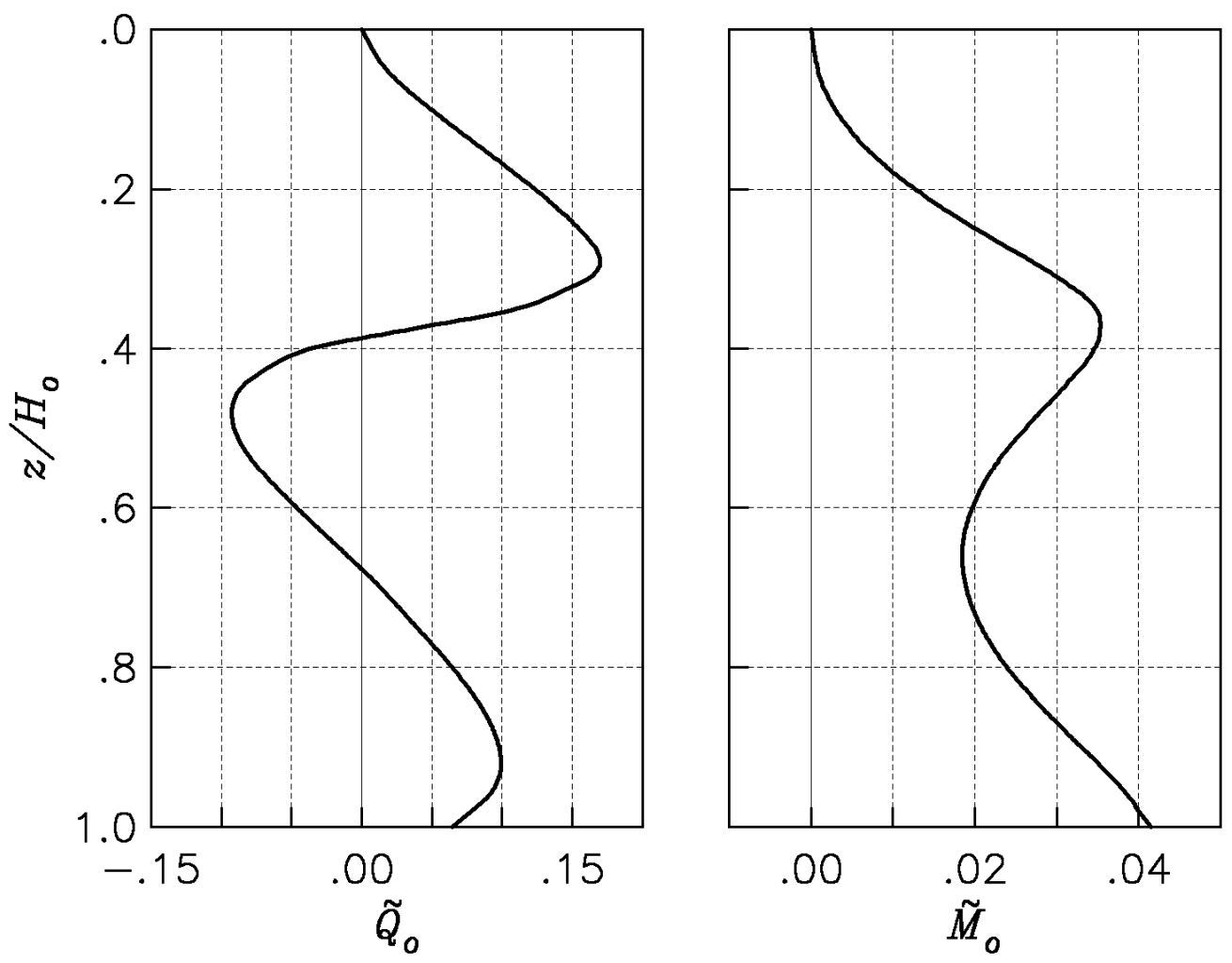
Tabla 8. Coeficientes de respuesta para el caso C1.

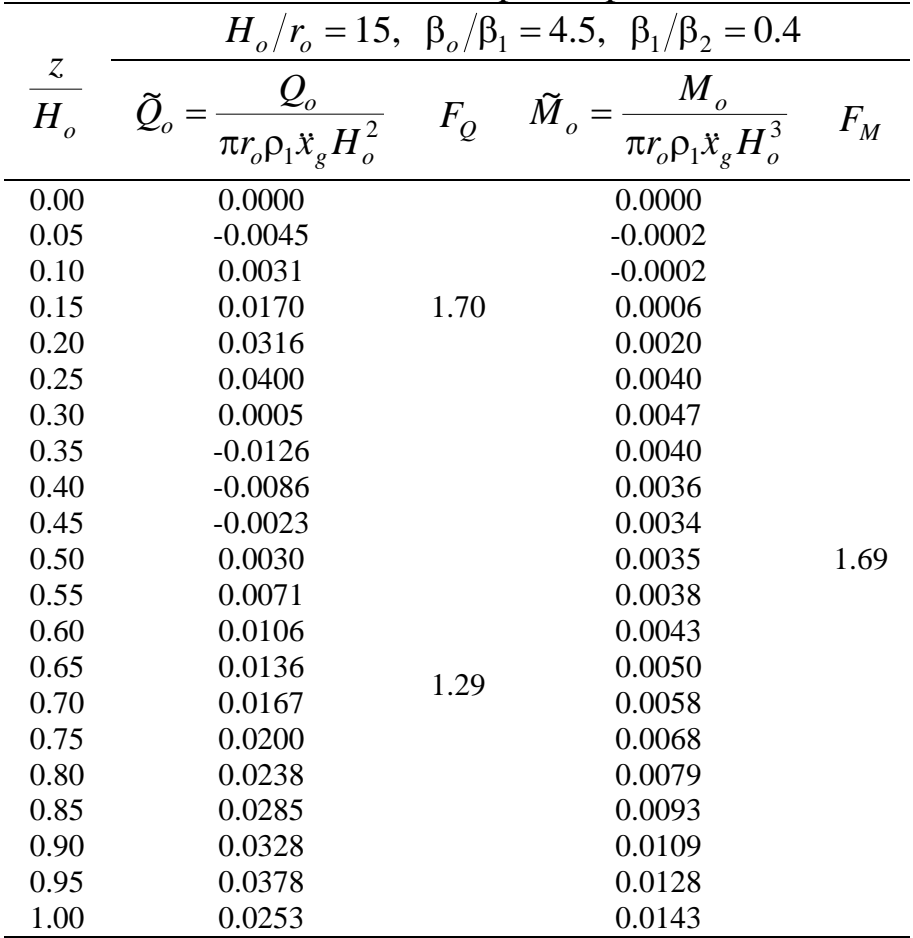
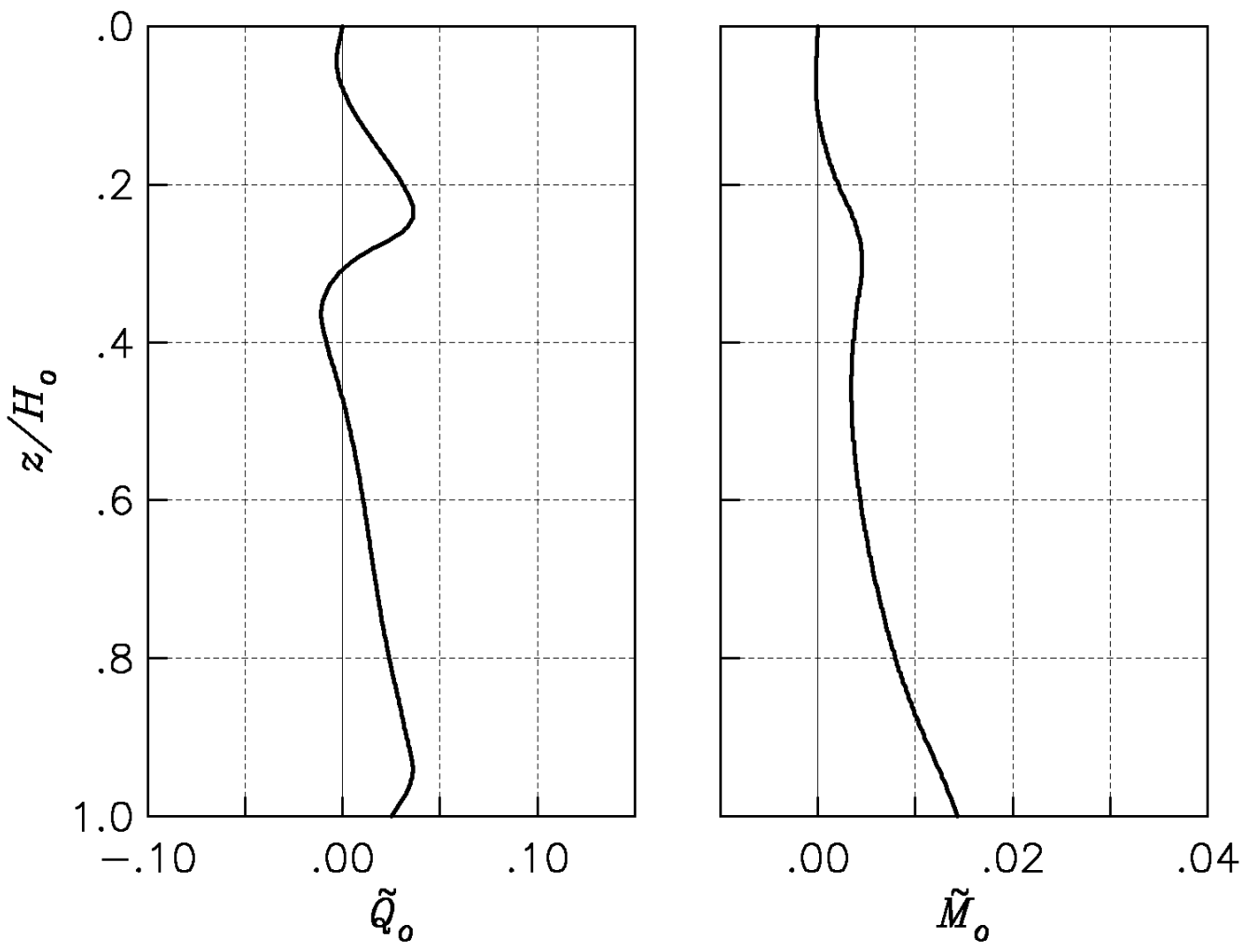
Tabla 9. Coeficientes de respuesta para el caso C2.

\begin{tabular}{|c|c|c|c|c|c|}
\hline \multirow[b]{2}{*}{$\frac{z}{H_{o}}$} & \multicolumn{5}{|c|}{$H_{o} / r_{o}=15, \quad \beta_{o} / \beta_{1}=9, \quad \beta_{1} / \beta_{2}=0.333$} \\
\hline & $\tilde{Q}_{o}=\frac{Q_{o}}{\pi r_{o} \rho_{1} x_{g} H_{o}^{2}}$ & $F_{Q}$ & $\tilde{M}_{o}=$ & $=\frac{M_{o}}{\pi r_{o} \rho_{1} x_{g} H_{o}^{3}}$ & $F_{M}$ \\
\hline 0.00 & 0.0000 & & & 0.0000 & \\
\hline 0.05 & -0.0001 & & & -0.0001 & \\
\hline 0.10 & 0.0148 & & & 0.0005 & \\
\hline 0.15 & 0.0360 & 1.90 & & 0.0022 & \\
\hline 0.20 & 0.0564 & & & 0.0048 & \\
\hline 0.25 & 0.0677 & & & 0.0081 & \\
\hline 0.30 & 0.0056 & & & 0.0095 & \\
\hline 0.35 & -0.0290 & & & 0.0081 & \\
\hline 0.40 & -0.0273 & & & 0.0066 & \\
\hline 0.45 & -0.0152 & & & 0.0058 & \\
\hline 0.50 & -0.0027 & & & 0.0055 & 1.80 \\
\hline 0.55 & 0.0080 & & & 0.0059 & \\
\hline 0.60 & 0.0170 & & & 0.0066 & \\
\hline 0.65 & 0.0249 & & & 0.0078 & \\
\hline 0.70 & 0.0325 & 1.31 & & 0.0094 & \\
\hline 0.75 & 0.0406 & & & 0.0114 & \\
\hline 0.80 & 0.0496 & & & 0.0137 & \\
\hline 0.85 & 0.0599 & & & 0.0167 & \\
\hline 0.90 & 0.0706 & & & 0.0201 & \\
\hline 0.95 & 0.0714 & & & 0.0237 & \\
\hline 1.00 & 0.0494 & & & 0.0265 & \\
\hline
\end{tabular}
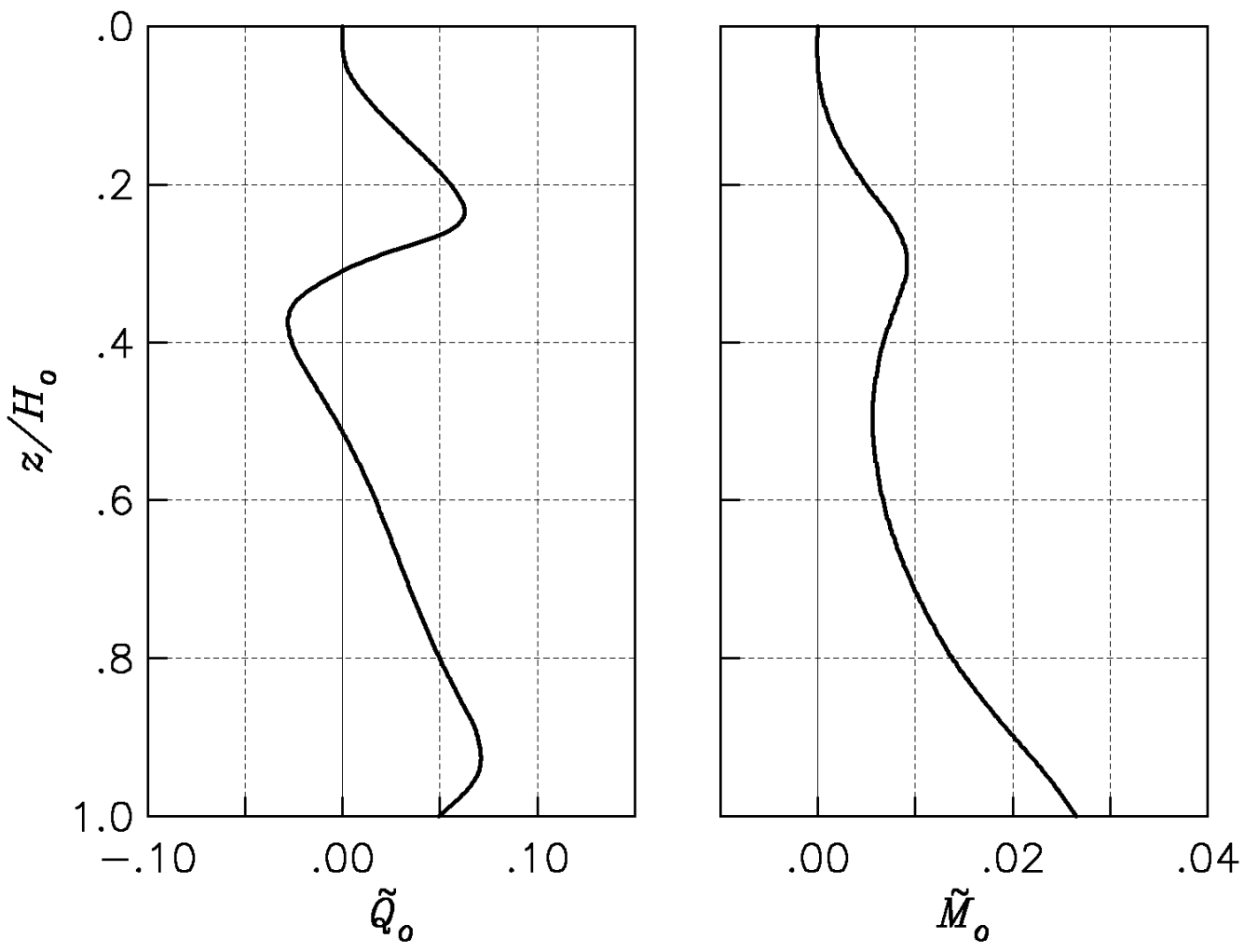
Tabla 10. Coeficientes de respuesta para el caso C3.

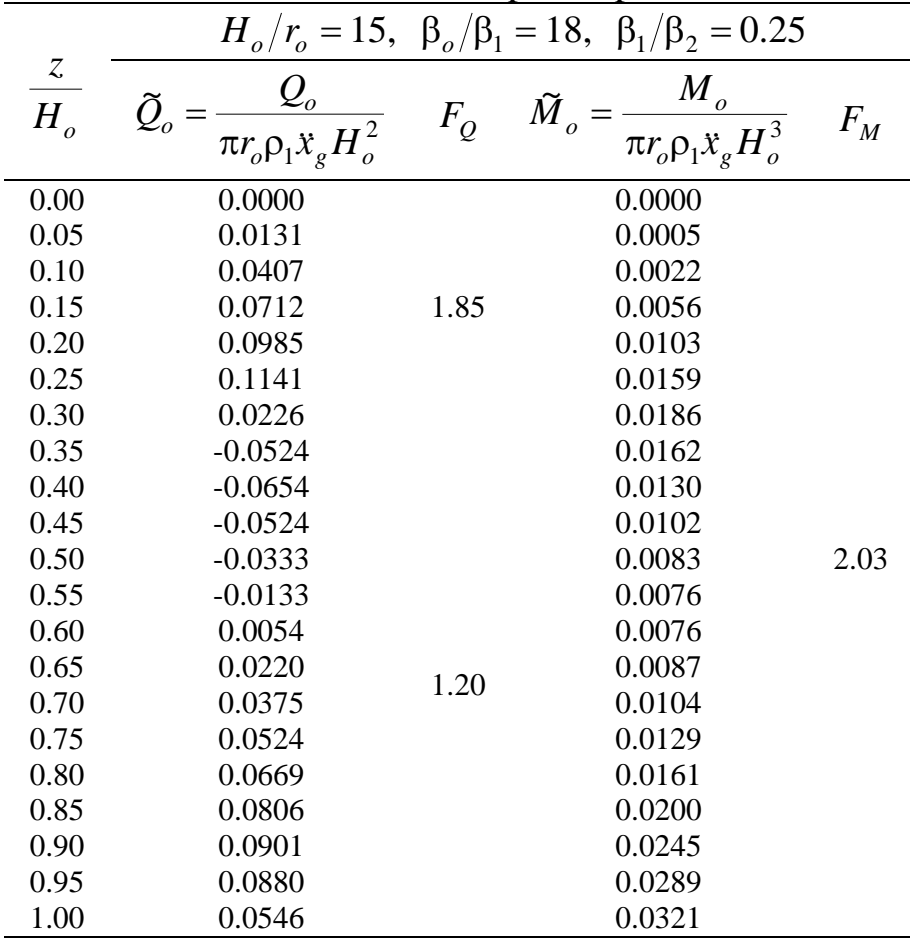
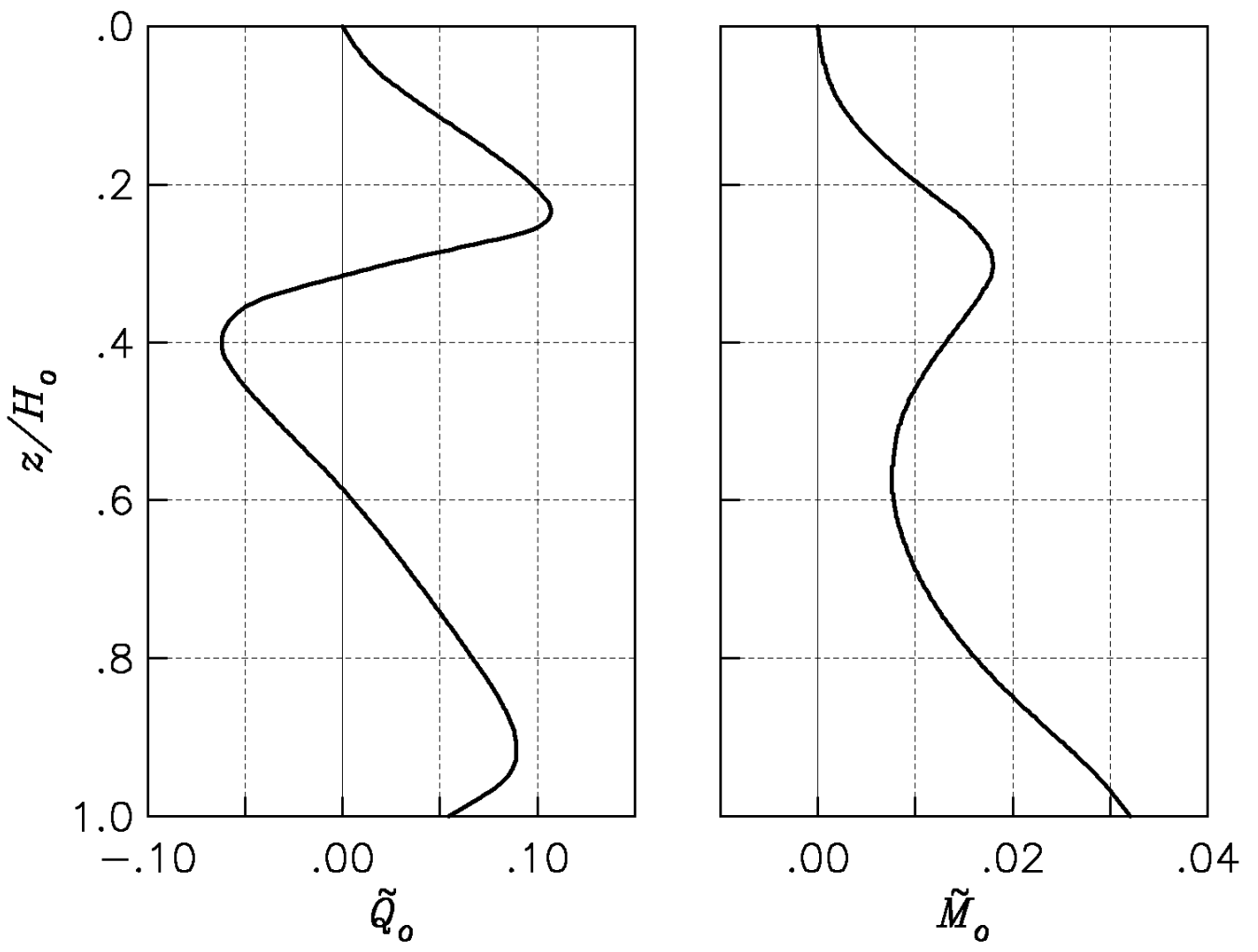
Los parámetros del sistema que se han variado son la relación de esbeltez $H_{o} / r_{o}$ y los contrastes de rigidez $\beta_{o} / \beta_{1}$ (estructura/suelo) y $\beta_{1} / \beta_{2}$ (suelo/basamento). En todos los casos analizados se supuso que $t=0.01 H_{o}$ (espesor del muro) y $d=0.02 H_{o}$ (peralte de la losa de fondo).

Se calcularon los valores estáticos del cortante y momento a diferente profundidad, así como los promedios de los valores dinámicos máximos que resultan de los sismos simulados. El cociente entre ellos define el correspondiente factor de amplificación dinámica. Estos resultados se presentan en las tablas 210 para diferentes relaciones de esbeltez y contrastes de rigidez. Usando estas tablas, los elementos mecánicos de diseño pueden calcularse siguiendo los siguientes pasos:

1. Para el sismo de diseño considerado, establecer la aceleración máxima en roca: $x_{g}(475)=91.5$ $\mathrm{cm} / \mathrm{s}^{2}$.

2. Calcular la velocidad de cortante y la densidad promedias del suelo hasta una profundidad $H_{1}=4 r_{o}$, mediante

$\beta_{1}, \rho_{1}=\frac{H_{1}}{\sum_{n} \frac{h_{n}}{\beta_{n}}, \frac{h_{n}}{\rho_{n}}}$

donde $\beta_{n}$ es la velocidad, $\rho_{n}$ la densidad y $h_{n}$ el espesor del $n$-ésimo estrato de los depósitos superficiales.

3. Determinar la relación de esbeltez $H_{o} / r_{o}$ y el contraste de rigidez estructura-suelo $\beta_{o} / \beta_{1}$, considerando que $\beta_{o}=\sqrt{G_{o} / \rho_{o}}$.

4. De las tablas 2-10, obtener el cortante y momento estáticos normalizados, $\widetilde{Q}_{o}$ y $\tilde{M}_{o}$, así como los correspondientes factores de amplificación dinámica, $F_{Q}$ y $F_{M}$. Para valores de $H_{o} / r_{o}$ y $\beta_{o} / \beta_{1}$ no tabulados, usar interpolación lineal.

5. Calcular los valores estáticos del cortante y momento como

$Q_{o}=\pi r_{o} \rho_{1} \ddot{x}_{g} H_{o}^{2} \widetilde{Q}_{o}$

$M_{o}=\pi r_{o} \rho_{1} x_{g} H_{o}^{3} \tilde{M}_{o}$

6. Para fines de diseño, determinar las acciones sísmicas como

$$
\begin{gathered}
Q_{d}=\frac{F_{Q} Q_{o}}{R \mu} \\
M_{d}=\frac{F_{M} M_{o}}{R \mu}
\end{gathered}
$$


donde $\mu=2$ es el factor de reducción por ductilidad y $R=1.5$ el factor de reducción por sobrerresistencia.

Para ilustrar el cálculo del cortante y momento basales, se analiza un sistema suelo-lumbrera con los siguientes datos:

- $\quad$ Altura de la lumbrera, $H_{o}=96 \mathrm{~m}$.

- $\quad$ Radio exterior de la lumbrera, $r_{o}=8 \mathrm{~m}$.

- Velocidad de cortante de la lumbrera, $\beta_{o}=2250 \mathrm{~m} / \mathrm{s}$.

- $\quad$ Velocidad de cortante y densidad promedias de los depósitos superficiales, $\beta_{1}=250 \mathrm{~m} / \mathrm{s}$ y $\rho_{1}=0.153$ ton $-\mathrm{s}^{2} / \mathrm{m}^{4}$.

Como $H_{o} / r_{o}=12$ y $\beta_{o} / \beta_{1}=9$, se usa la tabla 6 para el caso B2. Para $\mathrm{z} / H_{o}=1$, el cortante $\mathrm{y}$ momento estáticos normalizados, así como los correspondientes factores de amplificación dinámica, son iguales a:

$\widetilde{Q}_{o}=0.0571 \quad F_{Q}=1.26$

$\tilde{M}_{o}=0.0334 \quad F_{M}=1.77$

Las acciones sísmicas de diseño están dadas por:

$Q_{o}=\pi r_{o} \rho_{1} \ddot{x}_{g} H_{o}^{2} \tilde{Q}_{o}=\pi \times 8 \times 0.153 \times 0.915 \times 96^{2} \times 0.0571=1852$ ton

$Q_{d}=\frac{F_{Q} Q_{o}}{R \mu}=\frac{1.26 \times 1852}{1.5 \times 2}=778$ ton

$M_{o}=\pi r_{o} \rho_{1} \ddot{x}_{g} H_{o}^{3} \tilde{M}_{o}=\pi \times 8 \times 0.153 \times 0.915 \times 96^{3} \times 0.0334=103971$ ton $-m$

$M_{d}=\frac{F_{M} M_{o}}{R \mu}=\frac{1.77 \times 103971}{1.5 \times 2}=61343$ ton $-\mathrm{m}$

\section{CONCLUSIONES}

Se ha presentado un método de frontera para análisis sísmico dinámico de sistemas suelo-lumbrera, validado con resultados teóricos y prácticos. Los resultados calculados muestran que la flexibilidad del muro y la condición de apoyo tienen gran influencia en la magnitud y distribución de las acciones del suelo. En particular, el cortante y momento en lumbreras flotantes se reducen substancialmente comparados con los elementos mecánicos generados en lumbreras apoyadas o empotradas en el basamento. Para sistemas típicos excitados con sismos simulados, se construyeron tablas de diseño para calcular las distribuciones del cortante y momento con la profundidad. Para su aplicación se requiere del conocimiento de la aceleración máxima en roca y las características del sistema suelo-lumbrera. El procedimiento de diseño está basado en el cálculo de los valores estáticos del cortante y momento, 
multiplicados por factores de amplificación dinámica y reducidos por factores de ductilidad y sobrerresistencia.

El modelo presentado es elástico lineal. Para considerar efectos de no linealidad de los materiales, en una siguiente formulación se puede idealizar que el modelo está compuesto de tres regiones: una interior para representar la lumbrera, una central anular para representar al suelo circundante que se puede plastificar y una exterior para representar al suelo distante a la lumbrera, donde se pueden despreciar los efectos no lineales por interacción. Las dos regiones de suelo, en contacto mediante una superficie cilíndrica hipotética, se acoplan imponiendo la continuidad de esfuerzos y deformaciones. La rigidez y el amortiguamiento de cada estrato de suelo en cada región (exterior y central), se ajustan al nivel de deformaciones experimentado haciendo uso del método lineal equivalente. Con este modelo también se puede examinar la no linealidad en el material de la lumbrera. Este modelo puede complementarse incorporando una cuarta región en el interior de la lumbrera para simular el espacio hueco. Esto se lograría haciendo nulo el esfuerzo normal a la pared interior de la lumbrera.

\section{REFERENCIAS}

Arias A, F J Sánchez-Sesma y E Ovando-Shelley E (1981), "A simplified elastic model for seismic analysis of earth-retaining structures with limited displacements", Int. Conf. on Recent Advances in Geotechnical Earthquake Engineering and Soil Dynamics, St. Louis, MO, pp. 235-240.

Hashash Y M A, J J Hook, B Schmidt y J I-C Yao (2001), "Seismic design and analysis of underground structures”, Tunnelling and Underground Space Technology, Vol. 16, pp. 247-293.

Kausel E, J M Roësset y G Waas (1975), “Dynamic analysis of footings on layered media”, Journal of the Engineering Mechanics Division, ASCE, Vol. 101, pp. 679-693.

Kausel E y J M Roësset (1975), "Dynamic stiffness of circular foundations”, Journal of the Engineering Mechanics Division, ASCE, Vol. 101, pp. 771-785.

Kausel E y J M Roësset (1977), "Semianalytic hyperelement for layered strata”, Journal of the Engineering Mechanics Division, ASCE, Vol. 103, pp. 569-588.

Lysmer J y L A Drake (1972), “A finite element method for seismology”, Methods in Computational Physics. Advances in Research and Applications, Ed. B A Bolt, Vol. 11: Surface Waves and Earth Oscillations, Academic Press.

Lysmer J y G Waas (1972), "Shear waves in plane infinite structures”, Journal of the Engineering Mechanics Division, ASCE, Vol. 98, pp. 85-105.

St. John C M y T F Zahrah (1987), “Aseismic design of underground structures”, Tunnelling and Underground Space Technology, Vol. 2, pp. 165-197.

Tajimi H (1969), "Dynamic analysis of a structure embedded in an elastic stratum”, Fourth World Conference on Earthquake Engineering, Santiago, Chile, pp. 53-69.

Tassoulas J L y E Kausel (1983), “Elements for the numerical analysis of wave motion in layered media”, International Journal for Numerical Methods in Engineering, Vol. 19, pp. 1005-1032.

Veletsos A S y A H Younan (1994), “Dynamic soil pressures on rigid cylindrical vaults”, Earthquake Engineering and Structural Dynamics, Vol. 23, pp. 645-669.

Veletsos A S y A H Younan (1995), "Dynamic modeling and response of rigid embedded cylinders”, Journal of Engineering Mechanics, ASCE, Vol. 121, pp. 1026-1035. 
Zeevaert L (1983), “Lumbrera sujeta a la acción sísmica”, VI Congreso Nacional de Ingeniería Sísmica, Puebla, pp. 431-440. 


\section{APÉNDICE I MATRICES DE ESTRATO}

Suponiendo interpolación lineal de desplazamientos, las matrices de estrato para elementos planos y axisimétricos son las siguientes:

\section{Elementos planos en deformación plana}

$$
\begin{aligned}
& \tilde{A}_{j}=\frac{h_{j}}{6}\left[\begin{array}{cccc}
2\left(\lambda_{j}+2 G_{j}\right) & 0 & \lambda_{j}+2 G_{j} & 0 \\
0 & 2 G_{j} & 0 & G_{j} \\
\lambda_{j}+2 G_{j} & 0 & 2\left(\lambda_{j}+2 G_{j}\right) & 0 \\
0 & G_{j} & 0 & 2 G_{j}
\end{array}\right] \\
& \widetilde{B}_{j}=\frac{1}{2}\left[\begin{array}{cccc}
0 & -\left(\lambda_{j}-G_{j}\right) & 0 & \lambda_{j}+G_{j} \\
\lambda_{j}-G_{j} & 0 & \lambda_{j}+G_{j} & 0 \\
0 & -\left(\lambda_{j}+G_{j}\right) & 0 & \lambda_{j}-G_{j} \\
-\left(\lambda_{j}+G_{j}\right) & 0 & -\left(\lambda_{j}-G_{j}\right) & 0
\end{array}\right] \\
& \widetilde{G}_{j}=\frac{1}{h_{j}}\left[\begin{array}{cccc}
G_{j} & 0 & -G_{j} & 0 \\
0 & \lambda_{j}+2 G_{j} & 0 & -\left(\lambda_{j}+2 G_{j}\right) \\
-G_{j} & 0 & G_{j} & 0 \\
0 & -\left(\lambda_{j}+2 G_{j}\right) & 0 & \lambda_{j}+2 G_{j}
\end{array}\right] \tilde{M}_{j}=\rho_{j} h_{j}\left[\begin{array}{cccc}
1 / 3 & 0 & 1 / 6 & 0 \\
0 & 1 / 3 & 0 & 1 / 6 \\
1 / 6 & 0 & 1 / 3 & 0 \\
0 & 1 / 6 & 0 & 1 / 3
\end{array}\right]
\end{aligned}
$$

\section{Elementos planos en cortante antiplano}

$$
\tilde{A}_{j}=G_{j} h_{j}\left[\begin{array}{ll}
1 / 3 & 1 / 6 \\
1 / 6 & 1 / 3
\end{array}\right] \quad \tilde{G}_{j}=\frac{G_{j}}{h_{j}}\left[\begin{array}{rr}
1 & -1 \\
-1 & 1
\end{array}\right] \quad \tilde{M}_{j}=\rho_{j} h_{j}\left[\begin{array}{ll}
1 / 3 & 1 / 6 \\
1 / 6 & 1 / 3
\end{array}\right]
$$

\section{Elementos axisimétricos}

$$
\tilde{A}_{j}=\frac{h_{j}}{6}\left[\begin{array}{cccccc}
2\left(\lambda_{j}+2 G_{j}\right) & 0 & 0 & \lambda_{j}+2 G_{j} & 0 & 0 \\
0 & 2 G_{j} & 0 & 0 & G_{j} & 0 \\
0 & 0 & 2 G_{j} & 0 & 0 & G_{j} \\
\lambda_{j}+2 G_{j} & 0 & 0 & 2\left(\lambda_{j}+2 G_{j}\right) & 0 & 0 \\
0 & G_{j} & 0 & 0 & 2 G_{j} & 0 \\
0 & 0 & G_{j} & 0 & 0 & 2 G_{j}
\end{array}\right]
$$




$$
\begin{aligned}
& \widetilde{D}_{j}=\frac{1}{2}\left[\begin{array}{cccccc}
0 & \lambda_{j} & 0 & 0 & -\lambda_{j} & 0 \\
-G_{j} & 0 & 0 & G_{j} & 0 & 0 \\
0 & 0 & 0 & 0 & 0 & 0 \\
0 & \lambda_{j} & 0 & 0 & -\lambda_{j} & 0 \\
-G_{j} & 0 & 0 & G_{j} & 0 & 0 \\
0 & 0 & 0 & 0 & 0 & 0
\end{array}\right] \\
& \widetilde{E}_{j}=\frac{G_{j} h_{j}}{3 r_{o}}\left[\begin{array}{cccccc}
2 & 0 & 0 & 1 & 0 & 0 \\
0 & 0 & 0 & 0 & 0 & 0 \\
0 & 0 & 2 & 0 & 0 & 1 \\
1 & 0 & 0 & 2 & 0 & 0 \\
0 & 0 & 0 & 0 & 0 & 0 \\
0 & 0 & 1 & 0 & 0 & 2
\end{array}\right] \quad \widetilde{N}_{j}=\frac{G_{j} h_{j}}{6 r_{o}}\left[\begin{array}{cccccc}
0 & 0 & 4 & 0 & 0 & 2 \\
0 & 2 & 0 & 0 & 1 & 0 \\
4 & 0 & 0 & 2 & 0 & 0 \\
0 & 0 & 2 & 0 & 0 & 4 \\
0 & 1 & 0 & 0 & 2 & 0 \\
2 & 0 & 0 & 4 & 0 & 0
\end{array}\right] \\
& \tilde{L}_{j}=\frac{2 G_{j} h_{j}}{3 r_{o}^{2}}\left[\begin{array}{rrrrrr}
2 & 0 & -2 & 1 & 0 & -1 \\
0 & 0 & 0 & 0 & 0 & 0 \\
-2 & 0 & 2 & -1 & 0 & 1 \\
1 & 0 & -1 & 2 & 0 & -2 \\
0 & 0 & 0 & 0 & 0 & 0 \\
-1 & 0 & 1 & -2 & 0 & 2
\end{array}\right] \quad \widetilde{Q}_{j}=\frac{G_{j}}{2 r_{o}}\left[\begin{array}{rrrrrr}
0 & 0 & 0 & 0 & 0 & 0 \\
1 & 0 & -1 & -1 & 0 & 1 \\
0 & 0 & 0 & 0 & 0 & 0 \\
0 & 0 & 0 & 0 & 0 & 0 \\
1 & 0 & -1 & -1 & 0 & 1 \\
0 & 0 & 0 & 0 & 0 & 0
\end{array}\right]
\end{aligned}
$$

